\title{
Hyperbolic Second Order Equations with Non-Regular Time Dependent Coefficients
}

\author{
Claudia Garetto \& Michael Ruzhansky*
}

Communicated by G. DAL Maso

\begin{abstract}
In this paper we study weakly hyperbolic second order equations with time dependent irregular coefficients. This means assuming that the coefficients are less regular than Hölder. The characteristic roots are also allowed to have multiplicities. For such equations, we describe the notion of a 'very weak solution' adapted to the type of solutions that exist for regular coefficients. The construction is based on considering Friedrichs-type mollifiers of coefficients and corresponding classical solutions, and their quantitative behaviour in the regularising parameter. We show that even for distributional coefficients, the Cauchy problem does have a very weak solution, and that this notion leads to classical or to ultradistributional solutions under conditions when such solutions also exist. In concrete applications, the dependence on the regularising parameter can be traced explicitly.
\end{abstract}

\section{Introduction}

In this paper we study equations of the type

$$
\partial_{t}^{2} u(t, x)-\sum_{i=1}^{n} b_{i}(t) \partial_{t} \partial_{x_{i}} u(t, x)-\sum_{i=1}^{n} a_{i}(t) \partial_{x_{i}}^{2} u(t, x)=0, \quad x \in \mathbb{R}^{n}, t \in[0, T],
$$

where the coefficients are real and $a_{i} \geqq 0$ for all $i=1, \ldots, n$. It follows that this equation is hyperbolic (but not necessarily strictly hyperbolic). This kind of equation appears in many physical phenomena where discontinuous or singular

* M. RuZHANSKY was supported by the EPSRC Leadership Fellowship EP/G007233/1 and by EPSRC Grant EP/K039407/1. 
entities are involved, for instance in the wave propagation in a layered medium. An example is the wave equation

$$
\partial_{t}^{2} u(t, x)-\sum_{i=1}^{n} a_{i}(t) \partial_{x_{i}}^{2} u(t, x)=0,
$$

where the coefficients $a_{i}$ are Heaviside or Delta functions. In particular, when $n=2$, we can have the equation

$$
\partial_{t}^{2} u(t, x)-H_{t_{0}, t_{1}}(t) \partial_{x_{1}}^{2} u(t, x)-\delta_{t_{2}} \partial_{x_{2}}^{2} u(t, x)=0,
$$

where $0<t_{0}<t_{1} \leqq t_{2} \leqq T, H_{t_{0}, t_{1}}$ is the jump function with $H_{t_{0}, t_{1}}(t)=0$ for $t<t_{0}$ and $t>t_{1}$ and $H_{t_{0}, t_{1}}(t)=1$ for $t_{0} \leqq t \leqq t_{1}$, and $\delta_{t_{2}}$ is the Delta function concentrated at $t_{2}$. In this paper we will use the expression "a real-valued distribution" for a distribution $u \in \mathcal{D}(\mathbb{R})$ such that $u(\varphi) \in \mathbb{R}$ for all real-valued test functions $\varphi$. Similarly, we will write $u \geqq 0$ if $u(\varphi) \geqq 0$ for all non-negative test functions $\varphi \geqq 0$.

This is clearly the case for the coefficients above.

We also note that if $a_{i} \geqq 0$ is a non-negative distribution, it has to be of order zero, that is a Radon measure. However, we will not be making explicit use of this fact, thus, the analysis is extendible to more general sums of the form $\sum_{i, j=1}^{n} a_{i, j}(t) \partial_{x_{i}} \partial_{x_{j}} u$ in (1). However, certain modifications are needed in this case, in particular in the way this equation is reduced to a (hyperbolic) system - such an extension will be addressed elsewhere.

As usual, we will often rewrite the Equation (1) using the notation $D_{t}=-\mathrm{i} \partial_{t}$ and $D_{x_{i}}=-\mathrm{i} \partial_{x_{i}}$. The well-posedness of the corresponding Cauchy problem

$$
\begin{gathered}
D_{t}^{2} u(t, x)-\sum_{i=1}^{n} b_{i}(t) D_{t} D_{x_{i}} u(t, x)-\sum_{i=1}^{n} a_{i}(t) D_{x_{i}}^{2} u(t, x)=0, \\
u(0, x)=g_{0}, \\
D_{t} u(0, x)=g_{1},
\end{gathered}
$$

has been studied by many authors in the case of regular coefficients. If the coefficients $a_{i}$ and $b_{i}$ are sufficiently regular we can refer to the fundamental paper by Colombini et al. [6], showing that even if the coefficients are smooth, the wellposedness of the Cauchy problem (3) can be expected to hold only in Gevrey spaces (see also [9] for the weakly hyperbolic case). In fact, the famous example of Colombini and Spagnolo [13] shows that even if all $b_{i}=0$ and all $a_{i}$ are smooth, the Cauchy problem (3) may not be distributionally well-posed due to multiplicities. On the other hand, if the operator (1) is strictly hyperbolic, it was shown in [6] that the Cauchy problem (3) may still be distributionally ill-posed if the coefficients are less regular, for example only Hölder. An example of non-uniqueness in weakly hyperbolic problems was given in [10].

These examples, already for the second order equations with time-dependent coefficients as in (3), show the following by now well-known qualitative facts: 
- if the coefficients are smooth and the equation is strictly hyperbolic, the Cauchy problem (3) is distributionally well-posed (of course, much more is known, but it is less important for our purposes here);

- if the coefficients are smooth but the equation has multiplicities, then the Cauchy problem (3) may be not distributionally well-posed. However, it becomes wellposed in the appropriate classes of ultradistributions (depending on additional properties of coefficients or characteristic roots);

- if the equation is strictly hyperbolic but the coefficients are only Hölder continuous, the Cauchy problem (3) may be not distributionally well-posed. However, it becomes well-posed in the appropriate classes of ultradistributions;

- if the coefficients of the equation are continuous (and not Hölder continuous), there may be no ultradistributional well-posedness. However, it may become well-posed in the space of Fourier hyperfunctions.

As we see in the above statements, if we want to continue having a well-posedness result, the reduction in regularity assumptions on the coefficients leads to the necessity of weakening the notion of a solution to the Cauchy problem and enlarging the allowed class of solutions.

A threshold between distributional and ultradistributional well-posedness for equations with time-dependent coefficients (on the level of $C^{\infty}$ and Gevrey wellposedness) in terms of the regularity of coefficients has been discussed by COLOMBINI et al. [8]. We note that for $x$-dependent coefficients the situation becomes even much more subtle: for example, while very general Gevrey well-posedness results are available for Gevrey coefficients (see, for example BRONSHTEIN [4] or NISHITANI [26]), the $C^{\infty}$ well-posedness of second order equations with smooth coefficients is heavily dependent on the geometry of characteristics (see, for example [2,29]). Again, most such results can be translated into distributional or ultradistributional well-posedness, but still for equations with smooth or Gevrey coefficients.

The aim of this paper is to analyse the Cauchy problem (3) under much weaker regularity assumptions on coefficients. The general goal of reducing the regularity of coefficients for evolution partial differential equations has both mathematical and physical motivations, and has been thoroughly discussed by GELFAND [15], to which we refer also for the philosophical discussion of this topic.

Before we proceed with our approach, let us mention that the Cauchy problem (3) for operators with irregular coefficients has history and motivation from specific applied sciences. For example, problems of this type appear in geophysical applications with delta-like sources and discontinuous or more irregular media (for example, fractal-type media occurs naturally in the upper crust of the Earth or in fractured rocks), see [25], and especially [19] for a more detailed discussion and further references in geophysics and in tomography. Such problems have been treated using microlocal constructions in the Colombeau algebras, see for example HöRMANN and DE HoOP $[19,20]$. If the coefficients are measurable such equations often fall within the scope of problems which can be handled by semigroup methods, as in KATO [22]. However, to the best of our knowledge, there are no approaches to problems with irregularities like those in (2), providing both a well-posedness statement and a relation to 'classical' solutions. 
In this paper, we will look at the Cauchy problem (3) in different settings, the most general being that the coefficients $a_{i}$ and $b_{i}$ are distributions. In this case, in view of the famous Schwartz impossibility result on multiplication of distributions [30], the first question that arises is how to interpret the Equation (1) when $u$ is a distribution as well. A related question, for our purposes, is how to interpret the notion of a solution to the Cauchy problem (3). In view of the discussion above, it appears natural that in order to obtain solutions in this setting, one should weaken the notion of a solution to the Cauchy problem since ultradistributions or hyperfunctions may not be sufficient for such a purpose.

The aim and the main results of this paper are to show that:

- one can introduce the notion of 'very weak solutions' to the Cauchy problem (3), based on regularising coefficients and the Cauchy data with certain adaptation of Friedrichs mollifiers. Then, one can show that very weak solutions exist even if the coefficients and the Cauchy data are (compactly supported) distributions (Theorem 2.6);

- if the coefficients are sufficiently regular, namely, if they are in the class $C^{2}$, the very weak solutions all coincide in a certain sense, and are related to (coincide with) other known solutions. More precisely, if the Cauchy data are Gevrey ultradifferentiable functions, any very weak solution (for any regularisation of the coefficients) converges in the strong sense to the classical solution in the limit of the regularisation parameter. If the Cauchy data are distributions, any very weak solution (for any regularisation of the coefficients) converges in the ultradistributional sense to the ultradistributional solution in the limit of the regularisation parameter. See Theorem 2.7 for a precise formulation.

The appearance of the class $C^{2}$ is due to the fact that since we do not assume that the equation is strictly hyperbolic, the $C^{2}$-regularity of coefficients does guarantee that the characteristic roots of (1) are Lipschitz, and hence we know that the Gevrey or ultradistributional well-posedness holds. In the case that the equation is strictly hyperbolic, the assertions above still hold if the coefficients are, for example Lipschitz. Some further refinements are sometimes possible given precise relations between regularities of coefficients and roots of a hyperbolic polynomial (Bronshtein's theorem [3] and its refinements as in [12]).

The idea of considering regularisations of coefficients or solutions of hyperbolic partial differential equations in different senses is of course natural. For example, after regularising (for example non-Lipschitz, Hölder, etc.) coefficients with a parameter $\varepsilon$, relating $\varepsilon$ to some frequency zones in the energy estimate often yields the Gevrey or even $C^{\infty}$ well-posedness (see for example [6,7], and other papers). It is not always possible to relate $\varepsilon$ to frequency zones in which case families of solutions can be considered as a whole: for example, for hyperbolic equations with discontinuous coefficients, regularised families have been already considered by HURD and SATtinger [21], with a subsequent analysis of limits of these regularisations in $L^{2}$ as $\varepsilon \rightarrow 0$.

The purpose of this paper is to carry out a thorough analysis of appearing families of solutions and, by formulating a naturally associated notion of a 'very weak' 
solution, to relate it (as $\varepsilon \rightarrow 0$ ) to known classical, distributional or ultradistributional solutions.

In the next section we provide more specifics regarding the above statements. In particular, we briefly review the relevant ultradistributional well-posedness results, and put the notion of a very weak solution into a wider context.

In terms of the literature review for second order Cauchy problems (3), we will only give very specific references relevant to our subsequent purposes: for 'regular' coefficients much is known, for sharp results see, for e.g., Colombini et al. [6], NishitANi [26]. For references on equations with analytic coefficients, we refer to our recent paper [18]. Also, we do not discuss other interesting phenomena on the borderline of the existence of strong solutions (for example irregularity in $t$ can be sometimes compensated by favourable behaviour in $x$, see for example CICOGNANI and Colombini [5]).

\section{Main Results}

As we mentioned in the introduction, already when the coefficients are regular, there are several types of assumptions where we can expect qualitatively different results. On one hand, for very regular data, we may have well-posedness in the spaces of smooth, Gevrey, or analytic functions. At the duality level, this corresponds to the well-posedness in spaces of distributions, ultradistributions, or Fourier hyperfunctions.

We start by recalling the known results for coefficients which are regular: in [17], extending the one-dimensional result of KinOSHITA and SPAGNOLO in [23], we have obtained the following well-posedness result (for the special case of $b_{j}=0$, see also [9]):

\section{Theorem 2.1. [17]}

(i) If the coefficients $a_{j}, b_{j}, j=1, \ldots, n$, belong to $C^{k}([0, T])$ for some $k \geqq$ 2 and $g_{j} \in \gamma^{s}\left(\mathbb{R}^{n}\right)$ for $j=1,2$ then there exists a unique solution $u \in$ $C^{2}\left([0, T] ; \gamma^{s}\left(\mathbb{R}^{n}\right)\right)$ of the Cauchy problem (3) provided that

$$
1 \leqq s<1+\frac{k}{2}
$$

(ii) if the coefficients are of class $C^{\infty}$ on $[0, T]$ then the Cauchy problem (3) is well-posed in any Gevrey space;

(iii) under the hypotheses of (i), if the initial data $g_{j}$ are Gevrey Beurling ultradistributions in $\mathcal{E}_{(s)}^{\prime}\left(\mathbb{R}^{n}\right)$ for $j=1,2$ then there exists a unique solution $u \in C^{2}\left([0, T] ; \mathcal{D}_{(s)}^{\prime}\left(\mathbb{R}^{n}\right)\right)$ of the Cauchy problem (3) provided that

$$
1 \leqq s<1+\frac{k}{2}
$$

(iv) under the hypotheses of (ii) the Cauchy problem (3) is well-posed in any space of ultradistributions; 
(v) finally if the coefficients are analytic on $[0, T]$ then the Cauchy problem (3) is $C^{\infty}$ and distributionally well-posed.

For the sake of the reader we briefly recall the definitions of the spaces $\gamma^{s}\left(\mathbb{R}^{n}\right)$ and $\gamma^{(s)}\left(\mathbb{R}^{n}\right)$ of (Roumieu) Gevrey functions and (Beurling) Gevrey functions, respectively. These are intermediate classes between analytic functions $(s=1)$ and smooth functions. In the sequel, $\mathbb{N}_{0}=\{0,1,2, \ldots\}$.

Definition 2.2. Let $s \geqq 1$. We say that $f \in C^{\infty}\left(\mathbb{R}^{n}\right)$ belongs to the Gevrey (Roumieu) class $\gamma^{s}\left(\mathbb{R}^{n}\right)$ if for every compact set $K \subset \mathbb{R}^{n}$ there exists a constant $C>0$ such that for all $\alpha \in \mathbb{N}_{0}^{n}$ we have the estimate

$$
\sup _{x \in K}\left|\partial^{\alpha} f(x)\right| \leqq C^{|\alpha|+1}(\alpha !)^{s} .
$$

We say that $f \in C^{\infty}\left(\mathbb{R}^{n}\right)$ belongs to the Gevrey (Beurling) class $\gamma^{(s)}\left(\mathbb{R}^{n}\right)$ if for every compact set $K \subset \mathbb{R}^{n}$ and for every $A>0$ there exists a constant $C>0$ such that for all $\alpha \in \mathbb{N}_{0}^{n}$ we have the estimate

$$
\sup _{x \in K}\left|\partial^{\alpha} f(x)\right| \leqq C A^{|\alpha|+1}(\alpha !)^{s} .
$$

Let now $\gamma_{c}^{(s)}\left(\mathbb{R}^{n}\right)$ be the space of Beurling Gevrey functions with compact support. Its dual is the corresponding space $\mathcal{D}_{(s)}^{\prime}\left(\mathbb{R}^{n}\right)$ of ultradistributions and $\mathcal{E}_{(s)}^{\prime}\left(\mathbb{R}^{n}\right)$ is the subspace of compactly supported ultradistributions. We refer to [16] for relevant properties and Fourier characterisations of these spaces of ultradifferentiable functions and ultradistributions.

Going back to the Equation (1) and the corresponding Cauchy problem, we know that dropping the regularity assumption on the coefficients from $C^{k}$ to $C^{2 \alpha}$, with $\alpha \in(0,1]$, we still get Gevrey and ultradistributional well-posedness as proved for example in [11] for $n=1$, and in [16] for general $n$, which we follow:

Theorem 2.3. [16] Assume that the characteristic roots of the Equation (1) are of $\operatorname{class} C^{\alpha}, \alpha \in(0,1]$ in $t$.

(i) Let $g_{j} \in \gamma^{s}\left(\mathbb{R}^{n}\right)$ for $j=1,2$. Hence, the Cauchy problem (3) has a unique solution $u \in C^{2}\left([0, T], \gamma^{s}\left(\mathbb{R}^{n}\right)\right)$ provided that

$$
1 \leqq s<1+\alpha
$$

(ii) Let $g_{j} \in \mathcal{E}_{(s)}^{\prime}\left(\mathbb{R}^{n}\right)$ for $s=1,2$. Hence, the Cauchy problem (3) has a unique solution $u \in C^{2}\left([0, T], \mathcal{D}_{(s)}^{\prime}\left(\mathbb{R}^{n}\right)\right)$ provided that

$$
1 \leqq s \leqq 1+\alpha .
$$

(iii) If the roots are distinct then Gevrey and ultradistributional well-posedness hold provided that

$$
1 \leqq s<1+\frac{\alpha}{1-\alpha}
$$


and

$$
1 \leqq s \leqq 1+\frac{\alpha}{1-\alpha}
$$

respectively.

It is our purpose in this paper to prove the well-posedness of the Cauchy problem (3) when the coefficients are less than Hölder.

The first main idea now is to start from distributional coefficients $a_{i}$ and $b_{i}$, $i=1, \ldots, n$, to regularise them by convolution with a suitable mollifier $\psi$ obtaining families of smooth functions $\left(a_{i, \varepsilon}\right)_{\varepsilon}$ and $\left(b_{i, \varepsilon}\right)_{\varepsilon}$, namely

$$
a_{i, \varepsilon}=a_{i} * \psi_{\omega(\varepsilon)} \text { and } b_{i, \varepsilon}=b_{i} * \psi_{\omega(\varepsilon)},
$$

where

$$
\psi_{\omega(\varepsilon)}(t)=\omega(\varepsilon)^{-1} \psi(t / \omega(\varepsilon))
$$

and $\omega(\varepsilon)$ is a positive function converging to 0 as $\varepsilon \rightarrow 0$. It turns out that the nets $\left(a_{i, \varepsilon}\right)_{\varepsilon}$ and $\left(b_{i, \varepsilon}\right)_{\varepsilon}$ are $C^{\infty}$-moderate, in the sense that their $C^{\infty}$-seminorms can be estimated by a negative power of $\varepsilon$ (see Definition 2.4 and also (22)). More precisely, we will make use of the following notions of moderateness.

In the sequel, the notation $K \Subset \mathbb{R}^{n}$ means that $K$ is a compact set in $\mathbb{R}^{n}$.

Definition 2.4. (i) A net of functions $\left(f_{\varepsilon}\right)_{\varepsilon} \in C^{\infty}\left(\mathbb{R}^{n}\right)^{(0,1])}$ is $C^{\infty}$-moderate if for all $K \Subset \mathbb{R}^{n}$ and for all $\alpha \in \mathbb{N}_{0}^{n}$ there exist $N \in \mathbb{N}_{0}$ and $c>0$ such that

$$
\sup _{x \in K}\left|\partial^{\alpha} f_{\varepsilon}(x)\right| \leqq c \varepsilon^{-N}
$$

for all $\varepsilon \in(0,1]$.

(ii) A net of functions $\left(f_{\varepsilon}\right)_{\varepsilon} \in \gamma^{s}\left(\mathbb{R}^{n}\right)^{(0,1]}$ is $\gamma^{s}$-moderate if for all $K \Subset \mathbb{R}^{n}$ there exists a constant $c_{K}>0$ and there exists $N \in \mathbb{N}_{0}$ such that

$$
\left|\partial^{\alpha} f_{\varepsilon}(x)\right| \leqq c_{K}^{|\alpha|+1}(\alpha !)^{s} \varepsilon^{-N-|\alpha|},
$$

for all $\alpha \in \mathbb{N}_{0}^{n}, x \in K$ and $\varepsilon \in(0,1]$.

(iii) A net of functions $\left(f_{\varepsilon}\right)_{\varepsilon} \in C^{\infty}\left([0, T] ; \gamma^{s}\left(\mathbb{R}^{n}\right)\right)^{(0,1]}$ is $C^{\infty}\left([0, T] ; \gamma^{s}\left(\mathbb{R}^{n}\right)\right)$ moderate if for all $K \Subset \mathbb{R}^{n}$ there exist $N \in \mathbb{N}_{0}, c>0$ and, for all $k \in \mathbb{N}_{0}$ there exist $N_{k}>0$ and $c_{k}>0$ such that

$$
\left|\partial_{t}^{k} \partial_{x}^{\alpha} u_{\varepsilon}(t, x)\right| \leqq c_{k} \varepsilon^{-N_{k}} c^{|\alpha|+1}(\alpha !)^{s} \varepsilon^{-N-|\alpha|},
$$

for all $\alpha \in \mathbb{N}_{0}^{n}$, for all $t \in[0, T], x \in K$ and $\varepsilon \in(0,1]$.

We note that the conditions of moderateness are natural in the sense that regularisations of distributions or ultradistributions are moderate, namely we can think that

compactly supported distributions $\mathcal{E}^{\prime}\left(\mathbb{R}^{n}\right) \subset\left\{C^{\infty}\right.$-moderate families $\}$ 
by the structure theorems for distributions, while the regularisations of the compactly supported Gevrey ultradistributions can also be shown to be Gevrey-moderate.

Thus, while a solution to a Cauchy problem may not exist in the space on the left hand side of an inclusion like the one in (5), it may still exist (in a certain appropriate sense) in the space on its right hand side. The moderateness assumption will be enough for our purposes. However, we note that regularisation with standard Friedrichs mollifiers will not be sufficient, hence the introduction of a family $\omega(\varepsilon)$ in the above regularisations.

We can now introduce a notion of a 'very weak solution' for the Cauchy problem (3). We can also call it a Gevrey very weak solution (of order $s$ ), or simply a very weak solution.

Definition 2.5. Let $s \geqq 1$. The net $\left(u_{\varepsilon}\right)_{\varepsilon} \in C^{\infty}\left([0, T] ; \gamma^{s}\left(\mathbb{R}^{n}\right)\right)$ is a very weak solution of order $s$ of the Cauchy problem (3) if there exist

(i) $\quad C^{\infty}$-moderate regularisations $a_{i, \varepsilon}$ and $b_{i, \varepsilon}$ of the coefficients $a_{i}$ and $b_{i}$, respectively, for $i=1, \ldots, n$,

(ii) $\quad \gamma^{s}$-moderate regularisations $g_{0, \varepsilon}$ and $g_{1, \varepsilon}$ of the initial data $g_{0}$ and $g_{1}$, respectively,

such that $\left(u_{\varepsilon}\right)_{\varepsilon}$ solves the regularised problem

$$
\begin{gathered}
D_{t}^{2} u(t, x)-\sum_{i=1}^{n} b_{i, \varepsilon}(t) D_{t} D_{x_{i}} u(t, x)-\sum_{i=1}^{n} a_{i, \varepsilon}(t) D_{x_{i}}^{2} u(t, x)=0, \\
u(0, x)=g_{0, \varepsilon}, \\
D_{t} u(0, x)=g_{1, \varepsilon},
\end{gathered}
$$

for all $\varepsilon \in(0,1]$, and is $C^{\infty}\left([0, T] ; \gamma^{s}\left(\mathbb{R}^{n}\right)\right)$-moderate.

The main results of this paper can be summarised as the following solvability statement complemented by the uniqueness and consistency in Theorem 2.7.

Theorem 2.6. Let the coefficients $a_{i}, b_{i}$ of the Cauchy problem (3) be distributions with compact support included in $[0, T]$, such that $a_{i}, b_{i}$ are real-valued and $a_{i} \geqq 0$ for all $i=1, \ldots, n$. Let the Cauchy data $g_{0}, g_{1}$ be compactly supported distributions. Then, the Cauchy problem (3) has a very weak solution of order s, for all $s>1$.

In fact, Theorem 2.6 will be refined according to the regularity of the initial data. More precisely, we will distinguish between the following cases:

Case 1: distributional coefficients and Gevrey initial data;

Case 2: distributional coefficients and smooth initial data;

Case 3: distributional coefficients and distributional initial data.

The uniqueness and consistency result for very weak solutions of the Cauchy problem (3) is as follows. We distinguish between Gevrey Cauchy data and the general distributional Cauchy data: 
Theorem 2.7. Assume that the real-valued coefficients $a_{i}$ and $b_{i}$ are compactly supported, belong to $C^{k}([0, T])$ with $k \geqq 2$ and that $a_{i} \geqq 0$ for all $i=1, \ldots, n$. Let $1<s<1+\frac{k}{2}$.

- Let $g_{0}, g_{1} \in \gamma_{c}^{s}\left(\mathbb{R}^{n}\right)$. Then any very weak solution $\left(u_{\varepsilon}\right)_{\varepsilon}$ converges in the space $C\left([0, T] ; \gamma^{s}\left(\mathbb{R}^{n}\right)\right)$ as $\varepsilon \rightarrow 0$ to the unique classical solution in $C^{2}([0, T]$, $\left.\gamma^{s}\left(\mathbb{R}^{n}\right)\right)$. In particular, this limit exists and does not depend on the $C^{\infty}$ moderate regularisation of the coefficients.

- Let $g_{0}, g_{1} \in \mathcal{E}^{\prime}\left(\mathbb{R}^{n}\right)$. Then any very weak solution $\left(u_{\varepsilon}\right)_{\varepsilon}$ converges in the space $C\left([0, T] ; \mathcal{D}_{(s)}^{\prime}\left(\mathbb{R}^{n}\right)\right)$ to the unique solution in $C^{2}\left([0, T], \mathcal{D}_{(s)}^{\prime}\left(\mathbb{R}^{n}\right)\right)$. In particular, this limit exists and does not depend on the $C^{\infty}$-moderate regularisation of coefficients $a_{i}$ and $b_{i}$ and the Gevrey-moderate regularisation of the initial data $g_{0}, g_{1}$.

In Theorem 2.7, we assume that $1<s<1+\frac{k}{2}$ in order to make sure that the unique classical or ultradistributional solutions exist, provided by Theorem 2.1. Theorem 2.7 will follow from Theorem 7.1.

The proof of Theorem 2.6 relies on classical techniques for weakly hyperbolic equations (quasi-symmestriser, energy estimates, Gevrey-wellposedness, etc.) and ideas from generalised function theory (regularisation). In particular, proving the existence of a very weak solution coincides, by fixing the mollifiers, with proving well-posedness of the corresponding Cauchy problem in a suitable space of Colombeau type. This space will be chosen according to the regularity of the initial data. So, the proof of Theorem 2.6 will follow from the well-posedness results in Theorems 4.7, 5.3 and 6.3.

We note that the proof of Theorem 2.6 actually provides us with a description of possible regularisations, in particular, of functions $\omega(\varepsilon)$ used in the regularisation of coefficients in (4). Indeed, $\omega(\varepsilon)$ will be of the type $c\left(\log \left(\varepsilon^{-1}\right)\right)^{-r}$ or of the type $c\left(\log \left(\varepsilon^{-1}\right)\right)^{-r_{1}} \varepsilon^{-r_{2}}$, for $c>0$ and $r, r_{1}, r_{2}>0$.

We note that the idea of considering regularisations of coefficients and solutions of partial differential equations in different senses has been seen in the literature. For example, after regularising (for example non-Lipschitz, Hölder) coefficients with a parameter $\epsilon$, relating $\epsilon$ to some frequency zone in the energy estimate often yields the Gevrey or even $C^{\infty}$ well-posedness (see for example Colombini et al. [7] and other papers). For less regularity, for example for hyperbolic equations with discontinuous coefficients regularised families have been already considered by HuRD and SATTINGER [21], with a subsequent analysis of limits of these regularisations in $L^{2}$ as $\varepsilon \rightarrow 0$. An interesting result of well-posedness has been obtained for discontinuous and in general distributional coefficients in the Colombeau context by LAFON and Oberguggenberger [24]. In their paper they proved that first order symmetric systems of differential equations with Colombeau coefficients and Colombeau initial data have a unique Colombeau solution under suitable logarithmic type assumptions on the principal part. This result, while it can be easily extended to pseudo-differential systems, cannot be directly applied to our equation, since the system to which one can reduce our equations is, in general, non-symmetric and non-strictly hyperbolic. 
It will be useful also to us to use the developed machinery of Colombeau algebras in the proofs. In particular, this will provide an easy-to-get refinement of the uniqueness part of the corresponding statements. However, we need to work in algebras of generalised functions based on regularisations with Gevrey functions since smooth solutions do not have to exist due to multiplicities.

As mentioned above, we will employ quasi-symmetriser techniques, or more precisely, a parameterised version of the quasi-symmetriser seen in [17]. This is the topic of the next section.

\section{Parameter Dependent Quasi-Symmetriser}

In this paper, we will be applying the standard reduction of a scalar second order equation to the $2 \times 2$ system: setting

$$
u_{j}=D_{t}^{j-1}\left\langle D_{x}\right\rangle^{2-j} u, \quad j=1,2,
$$

we transform the equation

$$
D_{t}^{2} u(t, x)-\sum_{i=1}^{n} b_{i}(t) D_{t} D_{x_{i}} u(t, x)-\sum_{i=1}^{n} a_{i}(t) D_{x_{i}}^{2} u(t, x)=0
$$

into the hyperbolic system

$$
D_{t}\left(\begin{array}{l}
u_{1} \\
u_{2}
\end{array}\right)=\left(\begin{array}{cc}
0 & \left\langle D_{x}\right\rangle \\
\sum_{i=1}^{n} a_{i}(t) D_{x_{i}}^{2}\left\langle D_{x}\right\rangle^{-1} & \sum_{i=1}^{n} b_{i}(t) D_{x_{i}}
\end{array}\right)\left(\begin{array}{l}
u_{1} \\
u_{2}
\end{array}\right) .
$$

We now assume that the equation coefficients are distributions with compact support contained in $[0, T]$. Since the formulation of (1) might be impossible due to issues related to the product of distributions, we replace (1) with a regularised equation. In other words, we regularise every $a_{i}$ and $b_{i}$ by a convolution with a mollifier in $C_{c}^{\infty}\left(\mathbb{R}^{n}\right)$ and get nets of smooth functions as coefficients. More precisely, let $\psi \in C_{c}^{\infty}(\mathbb{R}), \psi \geqq 0$ with $\int \psi=1$ and let $\omega(\varepsilon)$ be a positive function converging to 0 as $\varepsilon \rightarrow 0$. Define

$$
\begin{aligned}
\psi_{\omega(\varepsilon)}(t) & :=\frac{1}{\omega(\varepsilon)} \psi\left(\frac{t}{\omega(\varepsilon)}\right), \\
a_{i, \varepsilon}(t) & :=\left(a_{i} * \psi_{\omega(\varepsilon)}\right)(t), \quad t \in[0, T]
\end{aligned}
$$

and

$$
b_{i, \varepsilon}(t):=\left(b_{i} * \psi_{\omega(\varepsilon)}\right)(t), \quad t \in[0, T] .
$$

By the structure theorem for compactly supported distributions, we have that there exists $L \in \mathbb{N}_{0}$ and $c>0$ such that

$$
\left|a_{i, \varepsilon}(t)\right| \leqq c \omega(\varepsilon)^{-L}, \quad\left|b_{i, \varepsilon}(t)\right| \leqq c \omega(\varepsilon)^{-L},
$$


for all $i=1, \ldots, n$. Regularising the Equation (1) means equivalently to regularise the system (6) as

$$
D_{t}\left(\begin{array}{l}
u_{1} \\
u_{2}
\end{array}\right)=\left(\begin{array}{cc}
0 & \left\langle D_{x}\right\rangle \\
\sum_{i=1}^{n} a_{i, \varepsilon}(t) D_{x_{i}}^{2}\left\langle D_{x}\right\rangle^{-1} & \sum_{i=1}^{n} b_{i, \varepsilon}(t) D_{x_{i}}
\end{array}\right)\left(\begin{array}{l}
u_{1} \\
u_{2}
\end{array}\right),
$$

with symbol matrix $A_{1, \varepsilon}(t, \xi)$. Clearly, one can write $A_{1, \varepsilon}(t, \xi)$ as $\langle\xi\rangle A_{\varepsilon}(t, \xi)$, where

$$
A_{\varepsilon}(t, \xi)=\left(\begin{array}{cc}
0 & 1 \\
\sum_{i=1}^{n} a_{i, \varepsilon}(t) \xi_{i}^{2}\langle\xi\rangle^{-2} & \sum_{i=1}^{n} b_{i, \varepsilon}(t) \xi_{i}\langle\xi\rangle^{-1}
\end{array}\right) .
$$

The matrix $A_{\varepsilon}(t, \xi)$ has eigenvalues

$$
\begin{aligned}
& \lambda_{1, \varepsilon}(t, \xi)=\frac{1}{2}\left(\sum_{i=1}^{n} b_{i, \varepsilon}(t) \xi_{i}\langle\xi\rangle^{-1}-\sqrt{\left(\sum_{i=1}^{n} b_{i, \varepsilon}(t) \xi_{i}\langle\xi\rangle^{-1}\right)^{2}+4 \sum_{i=1}^{n} a_{i, \varepsilon}(t) \xi_{i}^{2}\langle\xi\rangle^{-2}}\right) \\
& \lambda_{2, \varepsilon}(t, \xi)=\frac{1}{2}\left(\sum_{i=1}^{n} b_{i, \varepsilon}(t) \xi_{i}\langle\xi\rangle^{-1}+\sqrt{\left.\left(\sum_{i=1}^{n} b_{i, \varepsilon}(t) \xi_{i}\langle\xi\rangle^{-1}\right)^{2}+4 \sum_{i=1}^{n} a_{i, \varepsilon}(t) \xi_{i}^{2}\langle\xi\rangle^{-2}\right)} .\right.
\end{aligned}
$$

Note that $\lambda_{1, \varepsilon}\langle\xi\rangle$ and $\lambda_{2, \varepsilon}\langle\xi\rangle$ are the roots of the characteristic polynomial

$$
\tau^{2}-\sum_{i=1}^{n} b_{i, \varepsilon}(t) \xi_{i} \tau-\sum_{i=1}^{n} a_{i, \varepsilon}(t) \xi_{i}^{2}
$$

and fulfil the inequality

$$
\lambda_{1, \varepsilon}(t, \xi)^{2}+\lambda_{2, \varepsilon}(t, \xi)^{2} \leqq 2\left(\lambda_{1, \varepsilon}(t, \xi)-\lambda_{2, \varepsilon}(t, \xi)\right)^{2},
$$

employed by Kinoshita and Spagnolo in [23] to obtain Gevrey well-posedness for the corresponding Cauchy problem.

It is clear that the regularised Equation (1) and the corresponding first order system have solutions $\left(u_{\varepsilon}\right)_{\varepsilon}$ and $\left(U_{\varepsilon}\right)_{\varepsilon}$, respectively, depending on the parameter $\varepsilon \in(0,1]$. By Fourier transformation in $x$ the system

$$
D_{t} U_{\varepsilon}=\left(\begin{array}{cc}
0 & \left\langle D_{x}\right\rangle \\
\sum_{i=1}^{n} a_{i, \varepsilon}(t) D_{x_{i}}^{2}\left\langle D_{x}\right\rangle^{-1} & \sum_{i=1}^{n} b_{i, \varepsilon}(t) D_{x_{i}}
\end{array}\right) U_{\varepsilon},
$$

where

$$
U_{\varepsilon}=\left(\begin{array}{c}
u_{1, \varepsilon} \\
u_{2, \varepsilon}
\end{array}\right)=\left(\begin{array}{c}
\left\langle D_{x}\right\rangle u_{\varepsilon} \\
D_{t} u_{\varepsilon}
\end{array}\right)
$$

is transformed into

$$
D_{t} V_{\varepsilon}(t, \xi)=\langle\xi\rangle A_{\varepsilon}(t, \xi) V_{\varepsilon}(t, \xi)
$$

where

$$
V_{\varepsilon}(t, \xi)=\left(\mathcal{F} U_{\varepsilon}(t, \cdot)\right)(\xi)
$$


Finally, by regularising the initial data as well if needed (for instance in Case 3), we transform the Cauchy problem (3) into

$$
\begin{aligned}
D_{t} V_{\varepsilon}(t, \xi) & =\langle\xi\rangle A_{\varepsilon}(t, \xi) V_{\varepsilon}(t, \xi) \\
V_{0, \varepsilon} & =\left(\mathcal{F} U_{\varepsilon}(0, \cdot)\right)(\xi)=\mathcal{F}\left(\left\langle D_{x}\right\rangle g_{0, \varepsilon}, g_{1, \varepsilon}\right)
\end{aligned}
$$

The well-posedness of this regularised Cauchy problem will be obtained by constructing a quasi-symmetriser for the matrix $A_{\varepsilon}$ and the corresponding energy. Before proceeding with the technical details we recall some general basic facts. For more details see [14,23].

\subsection{The Quasi-Symmetriser: General Theory}

Note that for $m \times m$ matrices $A_{1}$ and $A_{2}$ the notation $A_{1} \leqq A_{2}$ means $\left(A_{1} v, v\right) \leqq$ $\left(A_{2} v, v\right)$ for all $v \in \mathbb{C}^{m}$ with $(\cdot, \cdot)$ the scalar product in $\mathbb{C}^{m}$.

Let $A(\lambda)$ be the $m \times m$ Sylvester matrix with real eigenvalues $\lambda_{l}$, that is,

$$
A(\lambda)=\left(\begin{array}{ccccc}
0 & 1 & 0 & \ldots & 0 \\
0 & 0 & 1 & \ldots & 0 \\
\ldots & \ldots & \ldots & \ldots & 1 \\
-\sigma_{m}^{(m)}(\lambda) & -\sigma_{m-1}^{(m)}(\lambda) & \ldots & \ldots & -\sigma_{1}^{(m)}(\lambda)
\end{array}\right),
$$

where

$$
\sigma_{h}^{(m)}(\lambda)=(-1)^{h} \sum_{1 \leqq i_{1}<\cdots<i_{h} \leqq m} \lambda_{i_{1}} \cdots \lambda_{i_{h}}
$$

for all $1 \leqq h \leqq m$. In the sequel we make use of the following notations: $\mathcal{P}_{m}$ for the class of permutations of $\{1, \ldots, m\}, \lambda_{\rho}=\left(\lambda_{\rho_{1}}, \ldots, \lambda_{\rho_{m}}\right)$ with $\lambda \in \mathbb{R}^{m}$ and $\rho \in \mathcal{P}_{m}, \pi_{i} \lambda=\left(\lambda_{1}, \ldots, \lambda_{i-1}, \lambda_{i+1}, \ldots, \lambda_{m}\right)$ and $\lambda^{\prime}=\pi_{m} \lambda=\left(\lambda_{1}, \ldots, \lambda_{m-1}\right)$. Following Section 4 in [23] we have that the quasi-symmetriser is the Hermitian matrix

$$
Q_{\delta}^{(m)}(\lambda)=\sum_{\rho \in \mathcal{P}_{m}} P_{\delta}^{(m)}\left(\lambda_{\rho}\right)^{*} P_{\delta}^{(m)}\left(\lambda_{\rho}\right),
$$

where $\delta \in(0,1], P_{\delta}^{(m)}(\lambda)=H_{\delta}^{(m)} P^{(m)}(\lambda), H_{\delta}^{(m)}=\operatorname{diag}\left\{\delta^{m-1}, \ldots, \delta, 1\right\}$ and the matrix $P^{(m)}(\lambda)$ is defined inductively by $P^{(1)}(\lambda)=1$ and

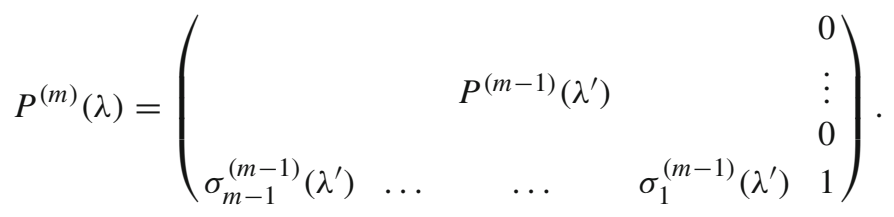

Note that $P^{(m)}(\lambda)$ is depending only on $\lambda^{\prime}$. Finally, let $W_{i}^{(m)}(\lambda)$ denote the row vector

$$
\left(\sigma_{m-1}^{(m-1)}\left(\pi_{i} \lambda\right), \ldots, \sigma_{1}^{(m-1)}\left(\pi_{i} \lambda\right), 1\right), \quad 1 \leqq i \leqq m
$$


and let $\mathcal{W}^{(m)}(\lambda)$ be the matrix with row vectors $W_{i}^{(m)}$. The following proposition collects the main properties of the quasi-symmetriser $Q_{\delta}^{(m)}(\lambda)$. For a detailed proof we refer the reader to Propositions 1 and 2 in [23] and to Proposition 1 in [14].

Proposition 3.1. (i) The quasi-symmetriser $Q_{\delta}^{(m)}(\lambda)$ can be written as

$$
Q_{0}^{(m)}(\lambda)+\delta^{2} Q_{1}^{(m)}(\lambda)+\cdots+\delta^{2(m-1)} Q_{m-1}^{(m)}(\lambda),
$$

where the matrices $Q_{i}^{(m)}(\lambda), i=1, \ldots, m-1$, are nonnegative and Hermitian with entries being symmetric polynomials in $\lambda_{1}, \ldots, \lambda_{m}$.

(ii) There exists a function $C_{m}(\lambda)$ bounded for bounded $|\lambda|$ such that

$$
C_{m}(\lambda)^{-1} \delta^{2(m-1)} I \leqq Q_{\delta}^{(m)}(\lambda) \leqq C_{m}(\lambda) I .
$$

(iii) We have

$$
\left|Q_{\delta}^{(m)}(\lambda) A(\lambda)-A(\lambda)^{*} Q_{\delta}^{(m)}(\lambda)\right| \leqq C_{m}(\lambda) \delta Q_{\delta}^{(m)}(\lambda)
$$

(iv) For any $(m-1) \times(m-1)$ matrix $T$ let $T^{\sharp}$ denote the $m \times m$ matrix

$$
\left(\begin{array}{ll}
T & 0 \\
0 & 0
\end{array}\right)
$$

Then, $Q_{\delta}^{(m)}(\lambda)=Q_{0}^{(m)}(\lambda)+\delta^{2} \sum_{i=1}^{m} Q_{\delta}^{(m-1)}\left(\pi_{i} \lambda\right)^{\sharp}$.

(v) We have

$$
Q_{0}^{(m)}(\lambda)=(m-1) ! \mathcal{W}^{(m)}(\lambda)^{*} \mathcal{W}^{(m)}(\lambda)
$$

(vi) We have

$$
\operatorname{det} Q_{0}^{(m)}(\lambda)=(m-1) ! \prod_{1 \leqq i<j \leqq m}\left(\lambda_{i}-\lambda_{j}\right)^{2} .
$$

(vii) There exists a constant $C_{m}$ such that

$$
q_{0,11}^{(m)}(\lambda) \cdots q_{0, m m}^{(m)}(\lambda) \leqq C_{m} \prod_{1 \leqq i<j \leqq m}\left(\lambda_{i}^{2}+\lambda_{j}^{2}\right) .
$$

We finally recall that a family $\left\{Q_{\alpha}\right\}$ of nonnegative Hermitian matrices is called nearly diagonal if there exists a positive constant $c_{0}$ such that

$$
Q_{\alpha} \geqq c_{0} \operatorname{diag} Q_{\alpha}
$$

for all $\alpha$, with

$$
\operatorname{diag} Q_{\alpha}=\operatorname{diag}\left\{q_{\alpha, 11}, \ldots, q_{\alpha, m m}\right\} \text {. }
$$

The following linear algebra result is proven in [23, Lemma 1]. 
Lemma 3.2. Let $\left\{Q_{\alpha}\right\}$ be a family of nonnegative Hermitian $m \times m$ matrices such that det $Q_{\alpha}>0$ and

$$
\text { det } Q_{\alpha} \geqq c q_{\alpha, 11} q_{\alpha, 22} \cdots q_{\alpha, m m}
$$

for a certain constant $c>0$ independent of $\alpha$. Then,

$$
Q_{\alpha} \geqq c m^{1-m} \operatorname{diag} Q_{\alpha}
$$

for all $\alpha$, that is, the family $\left\{Q_{\alpha}\right\}$ is nearly diagonal.

Lemma 3.2 is employed to prove that the family $Q_{\delta}^{(m)}(\lambda)$ of quasi-symmetrisers defined above is nearly diagonal when $\lambda$ belongs to a suitable set. The following statement is proven in [23, Proposition 3].

Proposition 3.3. For any $M>0$ define the set

$$
\mathcal{S}_{M}=\left\{\lambda \in \mathbb{R}^{m}: \lambda_{i}^{2}+\lambda_{j}^{2} \leqq M\left(\lambda_{i}-\lambda_{j}\right)^{2}, \quad 1 \leqq i<j \leqq m\right\}
$$

Then the family of matrices $\left\{Q_{\delta}^{(m)}(\lambda): 0<\delta \leqq 1, \lambda \in \mathcal{S}_{M}\right\}$ is nearly diagonal.

We conclude this section with a result on nearly diagonal matrices depending on three parameters (that is $\delta, t, \xi$ ) which will be crucial in the next section. Note that this is a straightforward extension of Lemma 2 in [23] valid for two parameter (that is $\delta, t$ ) dependent matrices.

Lemma 3.4. Let $\left\{Q_{\delta}^{(m)}(t, \xi): 0<\delta \leqq 1,0 \leqq t \leqq T, \xi \in \mathbb{R}^{n}\right\}$ be a nearly diagonal family of coercive Hermitian matrices of class $C^{k}$ in $t, k \geqq 1$. Then, there exists a constant $C_{T}>0$ such that for any non-zero continuous function $V:[0, T] \times \mathbb{R}^{n} \rightarrow \mathbb{C}^{m}$ we have

$$
\int_{0}^{T} \frac{\left|\left(\partial_{t} Q_{\delta}^{(m)}(t, \xi) V(t, \xi), V(t, \xi)\right)\right|}{\left(Q_{\delta}^{(m)}(t, \xi) V(t, \xi), V(t, \xi)\right)^{1-1 / k}|V(t, \xi)|^{2 / k}} \mathrm{~d} t \leqq C_{T}\left\|Q_{\delta}^{(m)}(\cdot, \xi)\right\|_{C^{k}([0, T])}^{1 / k}
$$

for all $\xi \in \mathbb{R}^{n}$.

\subsection{The Quasi-Symmetriser of the Matrix $A_{\varepsilon}$}

We now focus on the matrix $A_{\varepsilon}$ corresponding to the Cauchy problem we are studying. It is clear that we will get a family of quasi-symmetrisers $\left(Q_{\delta}^{(2)}\left(\lambda_{\varepsilon}\right)\right)_{\varepsilon}$, where $\lambda_{\varepsilon}=\left(\lambda_{1, \varepsilon}, \lambda_{2, \varepsilon}\right)$. More precisely, by direct computations we get

$$
Q_{\delta}^{(2)}\left(\lambda_{\varepsilon}\right)=\left(\begin{array}{cc}
\lambda_{1, \varepsilon}^{2}+\lambda_{2, \varepsilon}^{2} & -\left(\lambda_{1, \varepsilon}+\lambda_{2, \varepsilon}\right) \\
-\left(\lambda_{1, \varepsilon}+\lambda_{2, \varepsilon}\right) & 2
\end{array}\right)+2 \delta^{2}\left(\begin{array}{ll}
1 & 0 \\
0 & 0
\end{array}\right),
$$

where $\lambda_{1, \varepsilon}$ and $\lambda_{2, \varepsilon}$ are defined as in (7). Thus,

$$
\begin{aligned}
& Q_{\delta}^{(2)}\left(\lambda_{\varepsilon}\right)=\left(\begin{array}{cc}
\left(\sum_{i=1}^{n} b_{i, \varepsilon}(t) \xi_{i}\right)^{2}\langle\xi\rangle^{-2}+2 \sum_{i=1}^{n} a_{1, \varepsilon}(t) \xi_{i}^{2}\langle\xi\rangle^{-2} & -\sum_{i=1}^{n} b_{i, \varepsilon}(t) \xi_{i}\langle\xi\rangle^{-1} \\
-\sum_{i=1}^{n} b_{i, \varepsilon}(t) \xi_{i}\langle\xi\rangle^{-1} & 2
\end{array}\right) \\
& +2 \delta^{2}\left(\begin{array}{ll}
1 & 0 \\
0 & 0
\end{array}\right) \text {. }
\end{aligned}
$$


Note that from the formula (7), $\lambda_{1, \varepsilon}$ and $\lambda_{2, \varepsilon}$ are nets of smooth functions fulfilling the estimate

$$
\left|\partial_{t}^{(k)} \lambda_{i, \varepsilon}(t, \xi)\right| \leqq c_{k} \omega(\varepsilon)^{-L-k},
$$

for all $k \in \mathbb{N}_{0}$, for $t \in[0,1], \xi \in \mathbb{R}^{n}$ and $\varepsilon \in(0,1]$. Finally, since $\lambda_{1, \varepsilon}$ and $\lambda_{2, \varepsilon}$ in (7) are roots of a second order equation, they fulfil

$$
\lambda_{1, \varepsilon}^{2}(t, \xi)+\lambda_{2, \varepsilon}^{2}(t, \xi) \leqq 2\left(\lambda_{1, \varepsilon}(t, \xi)-\lambda_{2, \varepsilon}(t, \xi)\right)^{2},
$$

so the condition on the roots used in [23] and in [17] is trivially fulfilled with constant $M=2$ (see (6) in [17]).

By analysing Lemma 3.2 and Proposition 3.1 in this particular case we get the following results on the quasi-symmetriser $Q_{\delta}^{(2)}\left(\lambda_{\varepsilon}\right)$.

Proposition 3.5. Let $Q_{\delta}^{(2)}\left(\lambda_{\varepsilon}\right)$ as defined above. Then,

$$
\left(Q_{\delta}^{(2)}\left(\lambda_{\varepsilon}\right) V, V\right) \geqq \frac{1}{8}\left(Q_{\delta, \Delta}^{(2)}\left(\lambda_{\varepsilon}\right) V, V\right),
$$

where $Q_{\delta, \Delta}^{(2)}\left(\lambda_{\varepsilon}\right)$ is the diagonal part of the matrix $Q_{\delta}^{(2)}\left(\lambda_{\varepsilon}\right)$. In addition, there exists a constant $C_{2}>0$ such that

(i) $C_{2}^{-1} \omega(\varepsilon)^{2 L} \delta^{2} I \leqq Q_{\delta}^{(2)}\left(\lambda_{\varepsilon}(t, \xi)\right) \leqq C_{2} \omega(\varepsilon)^{-2 L} I$,

(ii) $\left|\left(\left(Q_{\delta}^{(2)}\left(\lambda_{\varepsilon}\right) A_{\varepsilon}(t, \xi)-A_{\varepsilon}(t, \xi)^{*} Q_{\delta}^{(2)}\left(\lambda_{\varepsilon}\right)\right) V, V\right)\right| \leqq C_{2} \delta\left(Q_{\delta}^{(2)}\left(\lambda_{\varepsilon}\right) V, V\right)$,

for all $\delta>0, \varepsilon \in(0,1], t \in[0, T], \xi \in \mathbb{R}^{n}$ and $V \in \mathbb{C}^{2}$.

Proof. By direct computations and by (11) we have that

$$
\begin{aligned}
\operatorname{det} Q_{\delta}^{(2)}\left(\lambda_{\varepsilon}\right) & =\left(\lambda_{1}-\lambda_{2}\right)^{2}+4 \delta^{2} \geqq \frac{1}{2}\left(\lambda_{1}(t, \xi)^{2}+\lambda_{2}(t, \xi)^{2}\right)+4 \delta^{2} \\
& \geqq \frac{2}{4}\left(\lambda_{1}(t, \xi)^{2}+\lambda_{2}(t, \xi)^{2}+2 \delta^{2}\right)=\frac{1}{4} q_{\delta, 11}\left(\lambda_{\varepsilon}\right) q_{\delta, 22}\left(\lambda_{\varepsilon}\right) .
\end{aligned}
$$

Note that the estimate below is uniform in $\varepsilon$ and $\delta$. Hence, Lemma 3.2 yields

$$
\left(Q_{\delta}^{(2)}\left(\lambda_{\varepsilon}\right) V, V\right) \geqq \frac{1}{8}\left(Q_{\delta, \Delta}^{(2)}\left(\lambda_{\varepsilon}\right) V, V\right) .
$$

We pass now to prove assertion (i). We have that

$$
\begin{aligned}
\left(Q_{\delta}^{(2)}\left(\lambda_{\varepsilon}\right) V, V\right) & =\left(\lambda_{1, \varepsilon}^{2}+\lambda_{2, \varepsilon}^{2}\right)\left|V_{1}\right|^{2}-2\left(\lambda_{1, \varepsilon}+\lambda_{2, \varepsilon}\right) \operatorname{Re}\left(\overline{V_{1}} V_{2}\right)+2 \delta^{2}\left|V_{1}\right|^{2}+2\left|V_{2}\right|^{2} \\
& =\left|\lambda_{1, \varepsilon} V_{1}-V_{2}\right|^{2}+\left|\lambda_{2, \varepsilon} V_{1}-V_{2}\right|^{2}+2 \delta^{2}\left|V_{1}\right|^{2} .
\end{aligned}
$$

It follows that if $\left|V_{1}\right|^{2} \geqq \gamma \omega(\varepsilon)^{2 L}\left|V_{2}\right|^{2}$, with $0 \leqq \gamma \leqq 1$ we have that $\left(Q_{\delta}^{(2)}\left(\lambda_{\varepsilon}\right) V, V\right) \geqq 2 \delta^{2}\left|V_{1}\right|^{2}=\delta^{2}\left(\left|V_{1}\right|^{2}+\left|V_{1}\right|^{2}\right) \geqq C_{2}^{-1} \omega(\varepsilon)^{2 L} \delta^{2}\left(\left|V_{1}\right|^{2}+\left|V_{2}\right|^{2}\right)$. 
On the other hand, recalling from (10) that $\left|\lambda_{i, \varepsilon}(t, \xi)\right|^{2} \omega(\varepsilon)^{2 L} \leqq c$ uniformly in variables and parameter for $i=1,2$, if $\left|V_{1}\right|^{2} \leqq \gamma \omega(\varepsilon)^{2 L}\left|V_{2}\right|^{2}$, we can write

$$
\begin{aligned}
\left(Q_{\delta}^{(2)}\left(\lambda_{\varepsilon}\right) V, V\right) & =\left|\lambda_{1, \varepsilon} V_{1}-V_{2}\right|^{2}+\left|\lambda_{2, \varepsilon} V_{1}-V_{2}\right|^{2}+2 \delta^{2}\left|V_{1}\right|^{2} \\
& \geqq \frac{1}{2}\left|V_{2}\right|^{2}-\lambda_{1, \varepsilon}^{2}\left|V_{1}\right|^{2}+\frac{1}{2}\left|V_{2}\right|^{2}-\lambda_{2, \varepsilon}^{2}\left|V_{1}\right|^{2}+2 \delta^{2}\left|V_{1}\right|^{2} \\
& \geqq\left|V_{2}\right|^{2}-\left(\lambda_{1, \varepsilon}^{2}+\lambda_{2, \varepsilon}^{2}\right) \gamma \omega(\varepsilon)^{2 L}\left|V_{2}\right|^{2}+2 \delta^{2}\left|V_{1}\right|^{2} \\
& \geqq\left(\left|V_{2}\right|^{2}-c \gamma\left|V_{2}\right|^{2}\right)+2 \delta^{2}\left|V_{1}\right|^{2} .
\end{aligned}
$$

So, choosing $\gamma$ sufficiently small and for $C_{2}$ big enough we have that

$$
\left(Q_{\delta}^{(2)}\left(\lambda_{\varepsilon}\right) V, V\right) \geqq C_{2}^{-1} \delta^{2}\left(\left|V_{1}\right|^{2}+\left|V_{2}\right|^{2}\right) .
$$

Combining (13) with (14) we conclude that

$$
\left(Q_{\delta}^{(2)}\left(\lambda_{\varepsilon}\right) V, V\right) \geqq C_{2}^{-1} \omega(\varepsilon)^{2 L} \delta^{2}\left(\left|V_{1}\right|^{2}+\left|V_{2}\right|^{2}\right),
$$

for all $V \in \mathbb{C}^{2}$. Finally, from (10) we have that

$$
\left(Q_{\delta}^{(2)}\left(\lambda_{\varepsilon}\right) V, V\right) \leqq C_{2} \omega(\varepsilon)^{-2 L}\left(\left|V_{1}\right|^{2}+\left|V_{2}\right|^{2}\right),
$$

proving in this way that assertion (i) holds.

We now want to prove assertion (ii). We begin by computing $Q_{\delta}^{(2)}\left(\lambda_{\varepsilon}\right) A_{\varepsilon}(t, \xi)-$ $A_{\varepsilon}(t, \xi)^{*} Q_{\delta}^{(2)}\left(\lambda_{\varepsilon}\right)$. We get

$$
Q_{\delta}^{(2)}\left(\lambda_{\varepsilon}\right) A_{\varepsilon}(t, \xi)-A_{\varepsilon}(t, \xi)^{*} Q_{\delta}^{(2)}\left(\lambda_{\varepsilon}\right)=\left(\begin{array}{lc}
0 & 2 \delta^{2} \\
-2 \delta^{2} & 0
\end{array}\right)
$$

and therefore

$$
\left(\left(Q_{\delta}^{(2)}\left(\lambda_{\varepsilon}\right) A_{\varepsilon}(t, \xi)-A_{\varepsilon}(t, \xi)^{*} Q_{\delta}^{(2)}\left(\lambda_{\varepsilon}\right)\right) V, V\right)=2 \delta^{2}\left(V_{2} \overline{V_{1}}-V_{1} \overline{V_{2}}\right)=4 i \delta^{2} \operatorname{Im} \overline{V_{1}} V_{2}
$$

This means that $\left(\left(Q_{\delta}^{(2)}\left(\lambda_{\varepsilon}\right) A_{\varepsilon}(t, \xi)-A_{\varepsilon}(t, \xi)^{*} Q_{\delta}^{(2)}\left(\lambda_{\varepsilon}\right)\right) V, V\right)$ does not depend on the eigenvalues $\lambda_{\varepsilon}=\left(\lambda_{1, \varepsilon}, \lambda_{2, \varepsilon}\right)$, or in other words, by replacing $Q_{\delta}^{(2)}\left(\lambda_{\varepsilon}\right)$ with $Q_{\delta, \Delta}^{(2)}\left(\lambda_{\varepsilon}\right)$ we can preliminary prove

$$
\begin{aligned}
& \left|\left(\left(Q_{\delta}^{(2)}\left(\lambda_{\varepsilon}\right) A_{\varepsilon}(t, \xi)-A_{\varepsilon}(t, \xi)^{*} Q_{\delta}^{(2)}\left(\lambda_{\varepsilon}\right)\right) V, V\right)\right| \\
& \quad=\left|\left(\left(Q_{\delta, \Delta}^{(2)}\left(\lambda_{\varepsilon}\right) A_{\varepsilon}(t, \xi)-A_{\varepsilon}(t, \xi)^{*} Q_{\delta, \Delta}^{(2)}\left(\lambda_{\varepsilon}\right)\right) V, V\right)\right| \leqq 2 \delta\left(Q_{\delta, \Delta}^{(2)}\left(\lambda_{\varepsilon}\right) V, V\right) .
\end{aligned}
$$

This is easily done. Indeed,

$$
\left|\left(\left(Q_{\delta}^{(2)}\left(\lambda_{\varepsilon}\right) A_{\varepsilon}(t, \xi)-A_{\varepsilon}(t, \xi)^{*} Q_{\delta}^{(2)}\left(\lambda_{\varepsilon}\right)\right) V, V\right)\right| \leqq 2 \delta 2 \delta\left|V_{1}\right|\left|V_{2}\right|
$$

and,

$$
\left(Q_{\delta, \Delta}^{(2)}\left(\lambda_{\varepsilon}\right) V, V\right) \geqq 2 \delta^{2}\left|V_{1}\right|^{2}+2\left|V_{2}\right|^{2} .
$$

It follows that

$$
2 \delta 2 \delta\left|V_{1}\right|\left|V_{2}\right| \leqq 2 \delta\left(\delta^{2}\left|V_{1}\right|^{2}+\left|V_{2}\right|^{2}\right),
$$


thus

$$
\left|\left(\left(Q_{\delta}^{(2)}\left(\lambda_{\varepsilon}\right) A_{\varepsilon}(t, \xi)-A_{\varepsilon}(t, \xi)^{*} Q_{\delta}^{(2)}\left(\lambda_{\varepsilon}\right)\right) V, V\right)\right| \leqq \delta\left(Q_{\delta, \Delta}^{(2)}\left(\lambda_{\varepsilon}\right) V, V\right) .
$$

The proof of assertion (ii) is completed by combining (16) with (12).

Note that

$$
\frac{1}{8}\left(Q_{\delta, \Delta}^{(2)}\left(\lambda_{\varepsilon}\right) V, V\right) \leqq\left(Q_{\delta}^{(2)}\left(\lambda_{\varepsilon}\right) V, V\right) \leqq 2\left(Q_{\delta, \Delta}^{(2)}\left(\lambda_{\varepsilon}\right) V, V\right) .
$$

Indeed,

$$
\begin{aligned}
\left(Q_{\delta}^{(2)}\left(\lambda_{\varepsilon}\right) V, V\right) & =\left(\lambda_{1, \varepsilon}^{2}+\lambda_{2, \varepsilon}^{2}\right)\left|V_{1}\right|^{2}-2\left(\lambda_{1, \varepsilon}+\lambda_{2, \varepsilon}\right) \operatorname{Re}\left(\overline{V_{1}} V_{2}\right)+2 \delta^{2}\left|V_{1}\right|^{2}+2\left|V_{2}\right|^{2} \\
& \leqq 2\left(\lambda_{1, \varepsilon}^{2}\left|V_{1}\right|^{2}+\left|V_{2}\right|^{2}\right)+2\left(\lambda_{2, \varepsilon}^{2}\left|V_{1}\right|^{2}+\left|V_{2}\right|^{2}\right)+2 \delta^{2}\left|V_{1}\right|^{2} \\
& \leqq 2\left(\lambda_{1, \varepsilon}^{2}+\lambda_{2, \varepsilon}^{2}+2 \delta^{2}\right)\left|V_{1}\right|^{2}+4\left|V_{2}\right|^{2}=2\left(Q_{\delta, \Delta}^{(2)}\left(\lambda_{\varepsilon}\right) V, V\right) .
\end{aligned}
$$

Adopting the notations of [23] we then have that the bound from below (19) in [23] is fulfilled with $c_{0}=\frac{1}{8}$. This means that the family of matrices

$$
\begin{aligned}
\left\{Q_{\delta, \varepsilon}^{(2)}(t, \xi)\right. & :=Q_{\delta}^{(2)}\left(\lambda_{\varepsilon}\right), \\
\lambda_{\varepsilon}(t, \xi) & \left.=\left(\lambda_{1, \varepsilon}(t, \xi), \lambda_{2, \varepsilon}(t, \xi)\right), t \in[0, T], \xi \in \mathbb{R}^{n}, \delta \in(0,1], \varepsilon \in(0,1]\right\}
\end{aligned}
$$

is nearly diagonal.

A careful analysis of the proof of Lemma 2 in [23] allows us to extend Lemma 3.4 to the family of quasi-symmetrisers $\left(Q_{\delta}^{(2)}\left(\lambda_{\varepsilon}\right)\right)_{\varepsilon}$. The constant $C_{T}=c_{0}^{-(1-1 / k)}$ in Lemma 2 is in our case equal to $(1 / 8)^{-(1-1 / k)}$.

Lemma 3.6. Let $\left\{Q_{\delta, \varepsilon}^{(2)}(t, \xi): 0<\delta \leqq 1,0<\varepsilon \leqq 1,0 \leqq t \leqq T, \xi \in \mathbb{R}^{n}\right\}$ be the nearly diagonal family of quasi-symmetrisers introduced above. Then, for any continuous function $V:[0, T] \times \mathbb{R}^{n} \rightarrow \mathbb{C}^{2}, V \neq 0$, we have

$$
\int_{0}^{T} \frac{\left|\left(\partial_{t} Q_{\delta, \varepsilon}^{(2)}(t, \xi) V(t, \xi), V(t, \xi)\right)\right|}{\left(Q_{\delta, \varepsilon}^{(2)}(t, \xi) V(t, \xi), V(t, \xi)\right)^{1-1 / k}|V(t, \xi)|^{2 / k}} \mathrm{~d} t \leqq C_{T}\left\|Q_{\delta, \varepsilon}^{(2)}(\cdot, \xi)\right\|_{C^{k}([0, T])}^{1 / k}
$$

for all $\xi \in \mathbb{R}^{n}, \delta \in(0,1]$ and $\varepsilon \in(0,1]$.

We are now ready to prove the well-posedness of the Cauchy problem (3). This will consist of two parts:

(i) choice of the framework,

(ii) energy estimates.

We begin by considering Case 1: distributional coefficients and Gevrey initial data.

\section{Case 1: Well-Posedness for Gevrey Initial Data}

We want to prove the well-posedness of the Cauchy problem (3) when the coefficients of the equation are distributions with compact support and the initial data are compactly supported Gevrey functions. This will be achieved in a suitable algebra of Colombeau type containing the usual Gevrey classes as subalgebras. We start by developing these objects. 


\subsection{Gevrey-Moderate Families}

We begin by investigating the convolution of a compactly supported Gevrey function with a mollifier $\varphi \in \mathscr{S}\left(\mathbb{R}^{n}\right)$ with $\int \varphi(x) \mathrm{d} x=1$ and $\int x^{\alpha} \varphi(x) \mathrm{d} x=0$ for all $\alpha \neq 0$ and

$$
\varphi_{\varepsilon}(x):=\varepsilon^{-n} \varphi(x / \varepsilon)
$$

The following holds:

Proposition 4.1. Let $\sigma>1$. Let $u \in \gamma_{c}^{\sigma}\left(\mathbb{R}^{n}\right)$ and let $\varphi$ be a mollifier as above. Then

(i) there exists $c>0$ such that

$$
\left|\partial^{\alpha}\left(u * \varphi_{\varepsilon}\right)(x)\right| \leqq c^{|\alpha|+1}(\alpha !)^{\sigma}
$$

for all $\alpha \in \mathbb{N}_{0}^{n}, x \in \mathbb{R}^{n}$ and $\varepsilon \in(0,1]$;

(ii) there exists $c>0$ and for all $q \in \mathbb{N}_{0}$ a constant $c_{q}>0$ such that

$$
\left|\partial^{\alpha}\left(u * \varphi_{\varepsilon}-u\right)(x)\right| \leqq c_{q} c^{|\alpha|+1}(\alpha !)^{\sigma} \varepsilon^{q},
$$

for all $\alpha \in \mathbb{N}_{0}^{n}, x \in \mathbb{R}^{n}$ and $\varepsilon \in(0,1]$;

(iii) there exist $c, c^{\prime}>0$ such that

$$
\left|\widehat{u * \varphi_{\varepsilon}}(\xi)\right| \leqq c^{\prime} \mathrm{e}^{-c\langle\xi\rangle^{\frac{1}{\sigma}}},
$$

for all $\xi \in \mathbb{R}^{n}$ and $\varepsilon \in(0,1]$.

Proof. (i) By convolution with the mollifier $\varphi_{\varepsilon}$ and straightforward estimates we obtain

$$
\left|\partial^{\alpha}\left(u * \varphi_{\varepsilon}\right)(x)\right|=\left|\partial^{\alpha} u * \varphi_{\varepsilon}(x)\right| \leqq \int_{\mathbb{R}^{n}}\left|\partial^{\alpha} u(x-\varepsilon z)\right||\varphi(z)| \mathrm{d} z \leqq c^{\mid \alpha++1}(\alpha !)^{\sigma},
$$

for all $\alpha \in \mathbb{N}_{0}^{n}, x \in \mathbb{R}^{n}$ and $\varepsilon \in(0,1]$.

(ii) Analogously, by Taylor expansion and the properties of the mollifier $\varphi$ (in particular since $\int x^{\alpha} \varphi(x) \mathrm{d} x=0$ for all $\alpha \neq 0$ ) we get for any $q \in \mathbb{N}_{0}$ the following estimate:

$$
\begin{aligned}
& \left|\partial^{\alpha}\left(u * \varphi_{\varepsilon}-u\right)(x)\right|=\left|\left(\partial^{\alpha} u * \varphi_{\varepsilon}-\partial^{\alpha} u\right)(x)\right| \\
& =\left|\int_{\mathbb{R}^{n}}\left(\partial^{\alpha} u(x-\varepsilon z)-\partial^{\alpha} u(x)\right) \varphi(z) \mathrm{d} z\right| \\
& =\left|\int_{\mathbb{R}^{n}} \sum_{|\beta|=q+1} \frac{\partial^{\alpha+\beta} u(x-\varepsilon \theta z)}{\beta !}(\varepsilon z)^{\beta} \varphi(z) \mathrm{d} z\right| \\
& \leqq \int_{\mathbb{R}^{n}} \sum_{|\beta|=q+1} \frac{\left|\partial^{\alpha+\beta} u(x-\varepsilon \theta z)\right|}{\beta !} \varepsilon^{q+1}\left|z^{\beta} \varphi(z)\right| \mathrm{d} z \\
& \leqq \varepsilon^{q+1} c^{|\alpha|+q+2} \sum_{|\beta|=q+1} \frac{((\alpha+\beta) !)^{\sigma}}{\beta !} \int_{\mathbb{R}^{n}} \sum_{|\beta|=q+1}\left|z^{\beta} \varphi(z)\right| \mathrm{d} z \\
& \leqq \varepsilon^{q+1} c^{|\alpha|+q+2} \sum_{|\beta|=q+1} \frac{2^{\sigma|\alpha|+\sigma|\beta|}(\alpha !)^{\sigma}(\beta !)^{\sigma}}{\beta !} \\
& \times \int_{\mathbb{R}^{n}} \sum_{|\beta|=q+1}\left|z^{\beta} \varphi(z)\right| \mathrm{d} z \leqq c_{q} \widetilde{c}^{|\alpha|+1}(\alpha !)^{\sigma} \varepsilon^{q} .
\end{aligned}
$$


Note that the estimate above holds for all $\alpha \in \mathbb{N}_{0}^{n}$ and $q \in \mathbb{N}_{0}$ uniformly in $x \in \mathbb{R}^{n}$ and $\varepsilon \in(0,1]$.

(iii) By Fourier transform we get that

$$
\widehat{u * \varphi_{\varepsilon}}(\xi)=\widehat{u}(\xi) \widehat{\varphi_{\varepsilon}}(\xi)=\widehat{u}(\xi) \widehat{\varphi}(\varepsilon \xi)
$$

and therefore since $u \in \gamma_{c}^{\sigma}\left(\mathbb{R}^{n}\right)$ and $\varphi \in \mathscr{S}\left(\mathbb{R}^{n}\right)$ the third assertion is trivial.

In Definition 2.4 we introduced the notion of a moderate net, that is, a net of functions $\left(f_{\varepsilon}\right)_{\varepsilon} \in \gamma^{\sigma}\left(\mathbb{R}^{n}\right)^{(0,1]}$ is $\gamma^{s}$-moderate if for all $K \Subset \mathbb{R}^{n}$ there exists a constant $c_{K}>0$ and there exists $N \in \mathbb{N}_{0}$ such that

$$
\left|\partial^{\alpha} f_{\varepsilon}(x)\right| \leqq c_{K}^{|\alpha|+1}(\alpha !)^{\sigma} \varepsilon^{-N-|\alpha|}
$$

for all $\alpha \in \mathbb{N}_{0}^{n}, x \in K$ and $\varepsilon \in(0,1]$.

Analogously one can talk of $\gamma^{\sigma}$-negligible nets.

Definition 4.2. Let $\sigma \geqq 1$. We say that $\left(u_{\varepsilon}\right)_{\varepsilon}$ is $\gamma^{\sigma}$-negligible if for all $K \Subset \mathbb{R}^{n}$ and for all $q \in \mathbb{N}_{0}$ there exists a constant $c_{q, K}>0$ such that

$$
\left|\partial^{\alpha} u_{\varepsilon}(x)\right| \leqq c_{q, K}^{|\alpha|+1}(\alpha !)^{\sigma} \varepsilon^{q-|\alpha|}
$$

for all $\alpha \in \mathbb{N}_{0}^{n}, x \in K$ and $\varepsilon \in(0,1]$.

We can now prove the following proposition.

Proposition 4.3. (i) If $\left(u_{\varepsilon}\right)_{\varepsilon}$ is $\gamma^{\sigma}$-moderate and there exists $K \Subset \mathbb{R}^{n}$ such that $\operatorname{supp} u_{\varepsilon} \subseteq K$ for all $\varepsilon \in(0,1]$ then there exist $c, c^{\prime}>0$ and $N \in \mathbb{N}_{0}$ such that

$$
\left|\widehat{u_{\varepsilon}}(\xi)\right| \leqq c^{\prime} \varepsilon^{-N} \mathrm{e}^{-c \varepsilon^{\frac{1}{\sigma}}\langle\xi\rangle \frac{1}{\sigma}}
$$

for all $\xi \in \mathbb{R}^{n}$.

(ii) If $\left(u_{\varepsilon}\right)_{\varepsilon}$ is $\gamma^{\sigma}$-negligible and there exists $K \Subset \mathbb{R}^{n}$ such that $\operatorname{supp} u_{\varepsilon} \subseteq K$ for all $\varepsilon \in(0,1]$ then there exists $c>0$ and for all $q>0$ there exists $c_{q}>0$ such that

$$
\left|\widehat{u_{\varepsilon}}(\xi)\right| \leqq c_{q} \varepsilon^{q} \mathrm{e}^{-c \varepsilon^{\frac{1}{\sigma}}\langle\xi\rangle^{\frac{1}{\sigma}}}
$$

for all $\xi \in \mathbb{R}^{n}$.

(iii) If $\left(u_{\varepsilon}\right)_{\varepsilon}$ is a net of tempered distributions with $\left(\widehat{u_{\varepsilon}}\right)_{\varepsilon}$ satisfying $(17)$ then $\left(u_{\varepsilon}\right)_{\varepsilon}$ is $\gamma^{s}$-moderate.

(iv) If $\left(u_{\varepsilon}\right)_{\varepsilon}$ is a net of tempered distributions with $\left(\widehat{u_{\varepsilon}}\right)_{\varepsilon}$ satisfying $(18)$ then $\left(u_{\varepsilon}\right)_{\varepsilon}$ is $\gamma^{s}$-negligible. 
Proof. (i) By elementary properties of the Fourier transform and since supp $u_{\varepsilon} \subseteq K$ for all $\varepsilon$ we have that

$$
\left|\xi^{\alpha} \widehat{u_{\varepsilon}}(\xi)\right|=\left|\mathcal{F}\left(D^{\alpha}\left(u_{\varepsilon}\right)\right)(\xi)\right| \leqq \int_{K}\left|\partial^{\alpha} u_{\varepsilon}(x)\right| \mathrm{d} x \leqq C^{|\alpha|+1}(\alpha !)^{\sigma} \varepsilon^{-|\alpha|-N},
$$

for all $\alpha \in \mathbb{N}_{0}^{n}$ and $\xi \in \mathbb{R}^{n}$. Let us now write $\langle\xi\rangle^{2 M}\left|\widehat{u_{\varepsilon}}(\xi)\right|^{2}$ as

$$
\sum_{k \leqq M}\left(\begin{array}{c}
M \\
k
\end{array}\right)|\xi|^{2 k}\left|\widehat{u_{\varepsilon}}(\xi)\right|^{2}=\sum_{k \leqq M}\left(\begin{array}{c}
M \\
k
\end{array}\right) \sum_{|\alpha| \leqq k} c_{\alpha} \xi^{2 \alpha}\left|\widehat{u_{\varepsilon}}(\xi)\right|^{2},
$$

where $c_{\alpha}>0$. Hence, from (19) we have

$$
\left|\xi^{\alpha} \widehat{u_{\varepsilon}}(\xi)\right|^{2} \leqq C^{2|\alpha|+2}(\alpha !)^{2 \sigma} \varepsilon^{-2|\alpha|-2 N}
$$

and, therefore, from $\alpha ! \leqq|\alpha|^{|\alpha|}$, we conclude

$$
\langle\xi\rangle^{2 M}\left|\widehat{u_{\varepsilon}}(\xi)\right|^{2} \leqq c_{M} C^{2 M+2} M^{2 \sigma M} \varepsilon^{-2 M-2 N} .
$$

It is clear that this last estimate implies

$$
\langle\xi\rangle^{M}\left|\widehat{u_{\varepsilon}}(\xi)\right| \leqq c_{M}^{\prime} C^{M+1} M^{\sigma M} \varepsilon^{-M-N},
$$

for all $M \in \mathbb{N}_{0}$, uniformly in $\varepsilon \in(0,1]$ and $\xi \in \mathbb{R}^{n}$. Note that by direct computations on the binomial coefficients one can see that the constant $c_{M}^{\prime}$ is of the type $C^{\prime M}$ so

$$
\langle\xi\rangle^{M}\left|\widehat{u_{\varepsilon}}(\xi)\right| \leqq C^{M+1} M^{\sigma M} \varepsilon^{-M-N} \leqq C^{M+1} \mathrm{e}^{\sigma M}(M !)^{\sigma} \varepsilon^{-M-N},
$$

for some suitable constant $C>0$. It follows that

$$
\langle\xi\rangle^{\frac{M}{\sigma}}\left|\widehat{u_{\varepsilon}}(\xi)\right|^{\frac{1}{\sigma}} \leqq C^{\frac{M}{\sigma}+\frac{1}{\sigma}} \mathrm{e}^{M} M ! \varepsilon^{-\frac{M}{\sigma}-\frac{N}{\sigma}} \leqq 2^{-M} C^{\frac{M}{\sigma}+\frac{1}{\sigma}} 2^{M} \mathrm{e}^{M} M ! \varepsilon^{-\frac{M}{\sigma}-\frac{N}{\sigma}},
$$

and therefore introducing a suitable constant $\nu>0$ (depending on $\sigma$ ) we have that

$$
\sum_{M}\left|\widehat{u_{\varepsilon}}(\xi)\right|^{\frac{1}{\sigma}}\left(v\langle\xi\rangle^{\frac{1}{\sigma}} \varepsilon^{\frac{1}{\sigma}}\right)^{M} \frac{1}{M !} \leqq \sum_{M} 2^{-M} \varepsilon^{-\frac{N}{\sigma}},
$$

for all $\xi \in \mathbb{R}^{n}$ and $\varepsilon>0$. In conclusion, recognising the Taylor series of an exponential in the previous formula, we arrive at

$$
\left|\widehat{u_{\varepsilon}}(\xi)\right| \leqq c^{\prime} \mathrm{e}^{-c\langle\xi\rangle^{\frac{1}{\sigma}} \varepsilon^{\frac{1}{\sigma}}} \varepsilon^{-N},
$$

for a suitable constants $c, c^{\prime}>0$ as desired.

(ii) The proof in (i) can be repeated for a $\gamma_{c}^{\sigma}$-negligible net $\left(u_{\varepsilon}\right)_{\varepsilon}$. From the assumption of negligibility it is immediate to see that the estimate

$$
\left|\widehat{u_{\varepsilon}}(\xi)\right| \leqq c_{q} \varepsilon^{q} \mathrm{e}^{-c \varepsilon^{\frac{1}{\sigma}}\langle\xi\rangle \frac{1}{\sigma}},
$$

holds uniformly in $\xi$ and $\varepsilon$. 
(iii) If $\left(u_{\varepsilon}\right)_{\varepsilon}$ is a net of tempered distributions satisfying (i) then by the Fourier characterisation of Gevrey functions $\left(u_{\varepsilon}\right)_{\varepsilon}$ is a net of Gevrey functions of order $\sigma$. More precisely,

$$
\begin{aligned}
\left|\partial^{\alpha} u_{\varepsilon}(x)\right| & =\left|\partial^{\alpha} \mathcal{F}^{-1}\left(\widehat{u_{\varepsilon}}\right)(x)\right| \leqq c \varepsilon^{-N} \int_{\mathbb{R}^{n}}\left|\xi^{\alpha}\right| \mathrm{e}^{-c \varepsilon^{\frac{1}{\sigma}}\langle\xi\rangle \frac{1}{\sigma}} \mathrm{d} \xi \\
& =c \varepsilon^{-N} \int_{\mathbb{R}^{n}} \mathrm{e}^{-\frac{c}{2} \varepsilon^{\frac{1}{\sigma}}\langle\xi\rangle^{\frac{1}{\sigma}}} \mathrm{d} \xi\left(\sup _{\xi \in \mathbb{R}^{n}}\left|\xi^{\alpha}\right| \mathrm{e}^{-\frac{c}{2} \varepsilon^{\frac{1}{\sigma}}\langle\xi\rangle \frac{1}{\sigma}}\right) \\
& \leqq c \varepsilon^{-N} \varepsilon^{-n} \int_{\mathbb{R}^{n}} \mathrm{e}^{-\frac{c}{2}|\xi|^{\frac{1}{\sigma}}} \mathrm{d} \xi\left(\sup _{\xi \in \mathbb{R}^{n}}\left|\xi^{\alpha}\right| \mathrm{e}^{-\frac{c}{2} \varepsilon^{\frac{1}{\sigma}}|\xi| \frac{1}{\sigma}}\right) \\
& \leqq c^{\prime} \varepsilon^{-N-n} \sup _{\xi \in \mathbb{R}^{n}}\left|\xi^{\alpha}\right| \mathrm{e}^{-\frac{c}{2} \varepsilon^{\frac{1}{\sigma}}\langle\xi\rangle} .
\end{aligned}
$$

Clearly,

$$
\sup _{|\xi| \leqq 1}\left|\xi^{\alpha}\right| \mathrm{e}^{-\frac{c}{2} \varepsilon^{\frac{1}{\sigma}}\langle\xi\rangle \frac{1}{\sigma}} \leqq 1 .
$$

Assume now that $|\xi| \geqq 1$. Hence

$$
\sup _{|\xi| \geqq 1}\left|\xi^{\alpha}\right| \mathrm{e}^{-\frac{c}{2} \varepsilon^{\frac{1}{\sigma}}\langle\xi\rangle^{\frac{1}{\sigma}}} \leqq \sup _{|\xi| \geqq 1}\left|\xi^{\alpha}\right| \mathrm{e}^{-\frac{c}{2} \varepsilon^{\frac{1}{\sigma}}|\xi| \frac{1}{\sigma}} .
$$

Note that there exists a constant $c_{\sigma}>0$ such that

$$
\begin{aligned}
\left|\xi^{\alpha}\right| \mathrm{e}^{-\frac{c}{2} \varepsilon^{\frac{1}{\sigma}}|\xi| \frac{1}{\sigma}} & =\varepsilon^{-|\alpha|}|\varepsilon \xi|^{\frac{|\alpha| \sigma}{\sigma}} \mathrm{e}^{-\frac{c}{2} \varepsilon^{\frac{1}{\sigma}}|\xi|^{\frac{1}{\sigma}}}=\varepsilon^{-|\alpha|}\left(|\varepsilon \xi|^{\mid \frac{|\alpha|}{\sigma}} \mathrm{e}^{-\frac{c}{2 \sigma}|\varepsilon \xi|^{\frac{1}{\sigma}}}\right)^{\sigma} \\
& =\varepsilon^{-|\alpha|}\left(\left(|\varepsilon \xi|^{\frac{1}{\sigma}} \frac{c}{2 \sigma}\right)^{|\alpha|} \mathrm{e}^{-\frac{c}{2 \sigma}|\varepsilon \xi|^{\frac{1}{\sigma}}}\right)^{\sigma}\left(\frac{c}{2 \sigma}\right)^{-|\alpha| \sigma} \\
& \leqq \varepsilon^{-|\alpha|}\left(\frac{c}{2 \sigma}\right)^{-|\alpha| \sigma}(|\alpha| !)^{\sigma} \\
& \leqq \varepsilon^{-|\alpha|}\left(\frac{c}{2 \sigma}\right)^{-|\alpha| \sigma} n^{|\alpha| \sigma}(\alpha !)^{\sigma} \leqq \varepsilon^{-|\alpha|} c_{\sigma}^{|\alpha|}(\alpha !)^{\sigma}
\end{aligned}
$$

Finally combining (20) with (21) we conclude that there exists a constant $C>0$ such that

$$
\left|\partial^{\alpha} u_{\varepsilon}(x)\right| \leqq C^{|\alpha|+1}(\alpha !)^{\sigma} \varepsilon^{-|\alpha|} \varepsilon^{-N-n},
$$

for all $x \in \mathbb{R}^{n}$ and $\varepsilon \in(0,1]$.

(iv) If $\left(u_{\varepsilon}\right)_{\varepsilon}$ is a net of tempered distributions satisfying $(i i)$ then by calculations analogous to the ones above (replacing $-N$ with $q$ ) we have that

$$
\left|\partial^{\alpha} u_{\varepsilon}(x)\right| \leqq C_{q}^{|\alpha|+1}(\alpha !)^{\sigma} \varepsilon^{q-n}
$$

for all $\varepsilon \in(0,1]$ and $x \in \mathbb{R}^{n}$. 
Making use of the previous definitions of $\gamma^{\sigma}$-moderate and negligible net (see Definition 4.2 and the paragraph above), we introduce the quotient space

$$
\mathcal{G}^{\sigma}\left(\mathbb{R}^{n}\right):=\frac{\gamma^{\sigma} \text {-moderate nets }}{\gamma^{\sigma} \text {-negligible nets }} .
$$

We now investigate the relationship between $\mathcal{G}^{\sigma}\left(\mathbb{R}^{n}\right)$ and the classical Colombeau algebra

$$
\mathcal{G}\left(\mathbb{R}^{n}\right)=\frac{\mathcal{E}_{M}\left(\mathbb{R}^{n}\right)}{\mathcal{N}\left(\mathbb{R}^{n}\right)}=\frac{C^{\infty} \text {-moderate nets }}{C^{\infty} \text {-negligible nets }} .
$$

We recall that a net $\left(u_{\varepsilon}\right)_{\varepsilon}$ is $C^{\infty}$-moderate if for all $K \Subset \mathbb{R}^{n}$ and all $\alpha \in \mathbb{N}_{0}^{n}$ there exist $c>0$ and $N \in \mathbb{N}_{0}$ such that

$$
\left|\partial^{\alpha} u_{\varepsilon}(x)\right| \leqq c \varepsilon^{-N},
$$

for all $x \in K$ and $\varepsilon \in(0,1]$. A net $\left(u_{\varepsilon}\right)_{\varepsilon}$ is $C^{\infty}$-negligible if for all $K \Subset \mathbb{R}^{n}$, all $\alpha \in \mathbb{N}_{0}^{n}$ and all $q \in \mathbb{N}_{0}$ there exists $c>0$ such that

$$
\left|\partial^{\alpha} u_{\varepsilon}(x)\right| \leqq c \varepsilon^{q},
$$

uniformly in $x \in K$ and $\varepsilon \in(0,1]$. For the general analysis of $\mathcal{G}\left(\mathbb{R}^{n}\right)$ we refer to for example OBERGUGGENBERGER [28].

Proposition 4.4. For all $\sigma \geqq 1$,

$$
\mathcal{G}^{\sigma}\left(\mathbb{R}^{n}\right) \subseteq \mathcal{G}\left(\mathbb{R}^{n}\right)
$$

Proof. To prove that $\mathcal{G}^{\sigma}\left(\mathbb{R}^{n}\right)$ is a subalgebra of $\mathcal{G}\left(\mathbb{R}^{n}\right)$ we need to prove that $\gamma^{\sigma}$-moderate and $\gamma^{\sigma}$-negligible nets are elements of $\mathcal{E}_{M}\left(\mathbb{R}^{n}\right)$ and $\mathcal{N}\left(\mathbb{R}^{n}\right)$, respectively and that if a $\gamma^{\sigma}$-moderate net belongs to $\mathcal{N}\left(\mathbb{R}^{n}\right)$ then it is automatically $\gamma^{\sigma}$ negligible. The first two implications are clear from the definition of $\gamma^{\sigma}$-moderate and $\gamma^{\sigma}$-negligible net. Finally, if $\left(u_{\varepsilon}\right)_{\varepsilon}$ is $\gamma^{\sigma}$-moderate and belongs to $\mathcal{N}\left(\mathbb{R}^{n}\right)$ then for all $K \Subset \mathbb{R}^{n}$ we have

$$
\left|\partial^{\alpha} u_{\varepsilon}(x)\right|^{2}=\left|\partial^{\alpha} u_{\varepsilon}(x)\right|\left|\partial^{\alpha} u_{\varepsilon}(x)\right| \leqq c_{K}^{|\alpha|+1}(\alpha !)^{\sigma} \varepsilon^{-N-|\alpha|} c_{K, q} \varepsilon^{q} .
$$

Choosing $q=2 q^{\prime}+N$ and by simple estimates we get

$$
\left|\partial^{\alpha} u_{\varepsilon}(x)\right|^{2} \leqq c_{K, q^{\prime}}^{2|\alpha|+2}(\alpha !)^{2 \sigma} \varepsilon^{2 q^{\prime}-2|\alpha|},
$$

which implies that the net $\left(u_{\varepsilon}\right)_{\varepsilon}$ is $\gamma^{\sigma}$-negligible.

The quotient space $\mathcal{G}^{\sigma}\left(\mathbb{R}^{n}\right)$ is a sheaf. This means that one can introduce a notion of restriction and a notion of support. More precisely, $x \in \mathbb{R}^{n} \backslash \operatorname{supp} u$ if there exists an open neighbourhood $V$ of $x$ such that $\left.u\right|_{V}=0$ in $\mathcal{G}^{\sigma}(V)$. Define $\mathcal{G}_{c}^{\sigma}\left(\mathbb{R}^{n}\right)$ as the algebra of compactly supported generalised functions in $\mathcal{G}^{\sigma}\left(\mathbb{R}^{n}\right)$. Making use of the previous arguments on $\gamma^{\sigma}$-moderate and -negligible nets we can prove the following proposition. 
Proposition 4.5. (i) If $u \in \mathcal{G}^{\sigma}\left(\mathbb{R}^{n}\right)$ has compact support then it has a representative $\left(u_{\varepsilon}\right)_{\varepsilon}$ and a compact set $K$ such that $\operatorname{supp} u_{\varepsilon} \subseteq K$ uniformly in $\varepsilon$.

(ii) $\gamma_{c}^{\sigma}\left(\mathbb{R}^{n}\right)$ is a subalgebra of $\mathcal{G}_{c}^{\sigma}\left(\mathbb{R}^{n}\right)$.

Proof. (i) We begin by recalling that if $u \in \mathcal{G}\left(\mathbb{R}^{n}\right)$ has compact support then it has a representative $\left(u_{\varepsilon}\right)_{\varepsilon}$ with $\operatorname{supp} u_{\varepsilon}$ contained in a compact set $K$ uniformly with respect to $\varepsilon$. In other words, there exists $\psi \in C_{c}^{\infty}\left(\mathbb{R}^{n}\right)$ identically one on a neighbourhood of supp $u$ such that $\psi u=u$ in $\mathcal{G}\left(\mathbb{R}^{n}\right)$. It follows that if $u \in \mathcal{G}^{\sigma}\left(\mathbb{R}^{n}\right)$ has compact support then $\psi u=u$ in $\mathcal{G}^{\sigma}\left(\mathbb{R}^{n}\right)$. Indeed,

$$
\left|\partial^{\alpha}\left(\psi u_{\varepsilon}\right)(x)\right| \leqq \sum_{\alpha^{\prime} \leqq \alpha}\left(\begin{array}{c}
\alpha \\
\alpha^{\prime}
\end{array}\right)\left|\partial^{\alpha^{\prime}} \psi(x)\right|\left|\partial^{\alpha-\alpha^{\prime}} u_{\varepsilon}(x)\right| \leqq c_{\psi} c_{\psi}^{|\alpha|+1}(\alpha !)^{\sigma} \varepsilon^{-N-|\alpha|} .
$$

This means that $\left(\psi u_{\varepsilon}\right)_{\varepsilon}$ is $\gamma_{c}^{\sigma}$-moderate. Since $\psi u_{\varepsilon}-u_{\varepsilon}$ is $\gamma^{\sigma}$-moderate and belongs to $\mathcal{N}\left(\mathbb{R}^{n}\right)$ as well, we conclude that $\left(\psi u_{\varepsilon}-u_{\varepsilon}\right)_{\varepsilon}$ is $\gamma^{\sigma}$-negligible.

(ii) The inclusion $\gamma_{c}^{\sigma}\left(\mathbb{R}^{n}\right) \subseteq \mathcal{G}_{c}^{\sigma}\left(\mathbb{R}^{n}\right)$ is a straightforward consequence of the fact that if $u \in \gamma_{c}^{\sigma}\left(\mathbb{R}^{n}\right)$ then $\left(u-u * \varphi_{\varepsilon}\right)_{\varepsilon}$ is $\gamma^{\sigma}$-negligible by Proposition 4.1 and $\operatorname{supp}\left[\left(u * \varphi_{\varepsilon}\right)_{\varepsilon}\right]=\operatorname{supp} u$.

An analogous version of Proposition 4.5 can be proven for $\mathcal{G}^{\sigma}\left(\mathbb{R}^{n}\right)$ and $\gamma^{\sigma}\left(\mathbb{R}^{n}\right)$, but it goes beyond the scope of this paper.

In this paper we will also make use of the following factor space.

Definition 4.6. Let $\left(u_{\varepsilon}(t, x)\right)_{\varepsilon} \in C^{\infty}\left([0, T] ; \gamma^{\sigma}\left(\mathbb{R}^{n}\right)\right)$. We say that the net $\left(u_{\varepsilon}\right)_{\varepsilon}$ is $C^{\infty}\left([0, T] ; \gamma^{\sigma}\left(\mathbb{R}^{n}\right)\right)$-moderate if for all $K \Subset \mathbb{R}^{n}$ there exist $N \in \mathbb{N}_{0}, c>0$ and, for all $k \in \mathbb{N}_{0}$ there exist $N_{k}>0$ and $c_{k}>0$ such that

$$
\left|\partial_{t}^{k} \partial_{x}^{\alpha} u_{\varepsilon}(t, x)\right| \leqq c_{k} \varepsilon^{-N_{k}} c^{|\alpha|+1}(\alpha !)^{\sigma} \varepsilon^{-N-|\alpha|},
$$

for all $\alpha \in \mathbb{N}_{0}^{n}$, for all $t \in[0, T], x \in K$ and $\varepsilon \in(0,1]$.

We say that the net $\left(u_{\varepsilon}\right)_{\varepsilon}$ is $C^{\infty}\left([0, T] ; \gamma^{\sigma}\left(\mathbb{R}^{n}\right)\right)$-negligible if for all $K \Subset \mathbb{R}^{n}$, for all $k \in \mathbb{N}_{0}$ and for all $q \in \mathbb{N}_{0}$ there exists $c>0$ such that

$$
\left|\partial_{t}^{k} \partial_{x}^{\alpha} u_{\varepsilon}(t, x)\right| \leqq c^{|\alpha|+1}(\alpha !)^{\sigma}{ }_{\varepsilon}^{q-|\alpha|}
$$

for all $\alpha \in \mathbb{N}_{0}^{n}$, for all $t \in[0, T], x \in K$ and $\varepsilon \in(0,1]$.

We denote the quotient space of $C^{\infty}\left([0, T] ; \gamma^{\sigma}\left(\mathbb{R}^{n}\right)\right)$-moderate nets with respect to $C^{\infty}\left([0, T] ; \gamma^{\sigma}\left(\mathbb{R}^{n}\right)\right)$-negligible nets by

$$
\mathcal{G}\left([0, T] ; \mathcal{G}^{\sigma}\left(\mathbb{R}^{n}\right)\right) .
$$

Note that the estimates in Definition 4.6 express the usual Colombeau properties in $t$ and the new Gevrey-Colombeau features in $x$ and that

$$
\mathcal{G}^{\sigma}\left(\mathbb{R}^{n}\right) \subseteq \mathcal{G}\left([0, T] ; \mathcal{G}^{\sigma}\left(\mathbb{R}^{n}\right)\right) \subseteq \mathcal{G}\left([0, T] \times \mathbb{R}^{n}\right)
$$

Moreover, in $\mathcal{G}\left([0, T] ; \mathcal{G}^{\sigma}\left(\mathbb{R}^{n}\right)\right)$ one can make use, at the level of representatives, of the characterisations by Fourier transform seen above (uniformly in $t \in[0, T]$ ). 


\subsection{Energy Estimate and Well-Posedness}

Let us define the energy

$$
E_{\delta, \varepsilon}(t, \xi):=\left(Q_{\delta, \varepsilon}^{(2)}(t, \xi) V(t, \xi), V(t, \xi)\right) .
$$

We have

$$
\begin{aligned}
\partial_{t} E_{\delta, \varepsilon}(t, \xi) & =\left(\partial_{t} Q_{\delta, \varepsilon}^{(2)} V, V\right)+i\left(Q_{\delta, \varepsilon}^{(2)} D_{t} V, V\right)-i\left(Q_{\delta, \varepsilon}^{(2)} V, D_{t} V\right) \\
& =\left(\partial_{t} Q_{\delta, \varepsilon}^{(2)} V, V\right)+i\left(Q_{\delta, \varepsilon}^{(2)} A_{1, \varepsilon} V, V\right)-i\left(Q_{\delta, \varepsilon}^{(2)} V, A_{1, \varepsilon} V\right) \\
& =\left(\partial_{t} Q_{\delta, \varepsilon}^{(2)} V, V\right)+i\langle\xi\rangle\left(\left(Q_{\delta, \varepsilon}^{(2)} A_{\varepsilon}-A_{\varepsilon}^{*} Q_{\delta, \varepsilon}^{(2)}\right) V, V\right) .
\end{aligned}
$$

It follows that

$$
\begin{aligned}
\partial_{t} E_{\delta, \varepsilon}(t, \xi) \leqq & \frac{\left|\left(\partial_{t} Q_{\delta, \varepsilon}^{(2)}(t, \xi) V(t, \xi), V(t, \xi)\right)\right| E_{\delta, \varepsilon}(t, \xi)}{\left(Q_{\delta, \varepsilon}^{(2)}(t, \xi) V(t, \xi), V(t, \xi)\right)} \\
& +\langle\xi\rangle\left|\left(\left(Q_{\delta, \varepsilon}^{(2)} A_{\varepsilon}-A_{\varepsilon}^{*} Q_{\delta, \varepsilon}^{(2)}\right)(t, \xi) V(t, \xi), V(t, \xi)\right)\right| .
\end{aligned}
$$

Now let

$$
K_{\delta, \varepsilon}(t, \xi):=\frac{\left|\left(\partial_{t} Q_{\delta, \varepsilon}^{(2)}(t, \xi) V(t, \xi), V(t, \xi)\right)\right|}{\left(Q_{\delta, \varepsilon}^{(2)}(t, \xi) V(t, \xi), V(t, \xi)\right)}
$$

provided that $V \neq 0$. Hence, we can rewrite (24) as

$$
\begin{aligned}
& \partial_{t} E_{\delta, \varepsilon}(t, \xi) \leqq K_{\delta, \varepsilon}(t, \xi) E_{\delta, \varepsilon}(t, \xi) \\
& \quad+\langle\xi\rangle\left|\left(\left(Q_{\delta, \varepsilon}^{(2)} A_{\varepsilon}-A_{\varepsilon}^{*} Q_{\delta, \varepsilon}^{(2)}\right)(t, \xi) V(t, \xi), V(t, \xi)\right)\right| .
\end{aligned}
$$

By Proposition 3.5(ii) we have that

$$
\begin{aligned}
\left|\left(\left(Q_{\delta, \varepsilon}^{(2)} A_{\varepsilon}-A_{\varepsilon}^{*} Q_{\delta, \varepsilon}^{(2)}\right)(t, \xi) V(t, \xi), V(t, \xi)\right)\right| & \left.\leqq C_{2} \delta\left(Q_{\delta, \varepsilon}^{(2)}\right)(t, \xi) V(t, \xi), V(t, \xi)\right) \\
& =C_{2} \delta E_{\delta, \varepsilon}(t, \xi) .
\end{aligned}
$$

Hence

$$
\partial_{t} E_{\delta, \varepsilon}(t, \xi) \leqq\left(K_{\delta, \varepsilon}(t, \xi)+C_{2} \delta\langle\xi\rangle\right) E_{\delta, \varepsilon}(t, \xi) .
$$

In the following we take any fixed integer $k \geqq 2$. Writing now

$$
\int_{0}^{T} K_{\delta, \varepsilon}(t, \xi) \mathrm{d} t
$$

as

$$
\int_{0}^{T} \frac{\left|\left(\partial_{t} Q_{\delta, \varepsilon}^{(2)}(t, \xi) V(t, \xi), V(t, \xi)\right)\right|}{\left(Q_{\delta, \varepsilon}^{(2)}(t, \xi) V(t, \xi), V(t, \xi)\right)^{1-1 / k}\left(Q_{\delta, \varepsilon}^{(2)}(t, \xi) V(t, \xi), V(t, \xi)\right)^{1 / k}} \mathrm{~d} t
$$


from the bound from below in Proposition 3.5(i), Lemma 3.6 and the estimates on the roots $\lambda_{i, \varepsilon}(t, \xi), i=1,2$, we have that

$$
\begin{aligned}
& \int_{0}^{T} K_{\delta, \varepsilon}(t, \xi) \mathrm{d} t \\
& \quad \leqq \int_{0}^{T} \frac{\left|\left(\partial_{t} Q_{\delta, \varepsilon}^{(2)}(t, \xi) V(t, \xi), V(t, \xi)\right)\right|}{\left(Q_{\delta, \varepsilon}^{(2)}(t, \xi) V(t, \xi), V(t, \xi)\right)^{1-1 / k}\left(C_{2}^{-1} \omega(\varepsilon)^{2 L} \delta^{2}|V(t, \xi)|^{2}\right)^{1 / k}} \mathrm{~d} t \\
& =C_{2}^{\frac{1}{k}} \delta^{-\frac{2}{k}} \omega(\varepsilon)^{-\frac{2 L}{k}} \int_{0}^{T} \frac{\left|\left(\partial_{t} Q_{\delta, \varepsilon}^{(2)}(t, \xi) V(t, \xi), V(t, \xi)\right)\right|}{\left(Q_{\delta, \varepsilon}^{(2)}(t, \xi) V(t, \xi), V(t, \xi)\right)^{1-1 / k}|V(t, \xi)|^{2 / k}} \mathrm{~d} t \\
& \leqq C_{2}^{\frac{1}{k}} \omega(\varepsilon)^{-\frac{2 L}{k}} \delta^{-\frac{2}{k}}\left\|Q_{\delta, \varepsilon}^{(2)}(\cdot, \xi)\right\|_{C^{k}([0, T])}^{1 / k} \leqq C_{1} \delta^{-\frac{2}{k}} \omega(\varepsilon)^{-\frac{2 L}{k}} \omega(\varepsilon)^{-\frac{L}{k}-1},
\end{aligned}
$$

uniformly in all the variables and parameters. Combining now (27) with the estimate on $\left|\left(\left(Q_{\delta, \varepsilon}^{(2)} A_{\varepsilon}-A_{\varepsilon}^{*} Q_{\delta, \varepsilon}^{(2)}\right)(t, \xi) V(t, \xi), V(t, \xi)\right)\right|$ above, by Gronwall lemma we obtain

$$
\begin{aligned}
E_{\delta, \varepsilon}(t, \xi) & \leqq E_{\delta, \varepsilon}(0, \xi) \mathrm{e}^{C_{1} \delta^{-\frac{2}{k}} \omega(\varepsilon)^{-\frac{3 L}{k}-1}+C_{2} T \delta\langle\xi\rangle} \\
& \leqq E_{\delta, \varepsilon}(0, \xi) \mathrm{e}^{C_{T}\left(\delta^{-\frac{2}{k}} \omega(\varepsilon)^{-\frac{3 L}{k}-1}+\delta\langle\xi\rangle\right)}
\end{aligned}
$$

As in [17] set $\delta^{-\frac{2}{k}}=\delta\langle\xi\rangle$. It follows that $\delta^{-\frac{2}{k}}=\langle\xi\rangle^{\frac{1}{\sigma}}$, where

$$
\sigma=1+\frac{k}{2}
$$

Making use of the estimates in Proposition 3.5(i), of the definition of $Q_{\delta, \varepsilon}^{(2)}$ and of the fact that $\omega(\varepsilon)^{-1} \geqq 1$, we obtain

$$
\begin{aligned}
C_{2}^{-1} \omega(\varepsilon)^{2 L} \delta^{2}|V(t, \xi)|^{2} & \leqq E_{\delta, \varepsilon}(t, \xi) \leqq E_{\delta, \varepsilon}(0, \xi) \mathrm{e}^{C_{T} \omega(\varepsilon)^{-\frac{3 L}{k}-1}\langle\xi\rangle^{\frac{1}{\sigma}}} \\
& \leqq C_{2} \omega(\varepsilon)^{-2 L}|V(0, \xi)|^{2} \mathrm{e}^{C_{T} \omega(\varepsilon)^{-\frac{3 L}{k}-1}\langle\xi\rangle^{\frac{1}{\sigma}}} .
\end{aligned}
$$

This implies, for $M=(3 L+k) / k$,

$$
\begin{aligned}
|V(t, \xi)|^{2} & \leqq C_{2}^{2} \delta^{-2} \omega(\varepsilon)^{-4 L}|V(0, \xi)|^{2} \mathrm{e}^{C_{T} \omega(\varepsilon)^{-M}\langle\xi\rangle^{\frac{1}{\sigma}}} \\
& =C_{2}^{2} \omega(\varepsilon)^{-4 L}\langle\xi\rangle^{\frac{k}{\sigma}}|V(0, \xi)|^{2} \mathrm{e}^{C_{T} \omega(\varepsilon)^{-M}\langle\xi\rangle^{\frac{1}{\sigma}}},
\end{aligned}
$$

or equivalently

$$
|V(t, \xi)| \leqq C \omega(\varepsilon)^{-2 L}\langle\xi\rangle^{\frac{k}{2 \sigma}}|V(0, \xi)| \mathrm{e}^{C \omega(\varepsilon)^{-M}\langle\xi\rangle^{\frac{1}{\sigma}}},
$$

for a suitable constant $C>0$.

We begin by assuming that the initial data are in $\gamma^{s}\left(\mathbb{R}^{n}\right)$. This means that

$$
|V(0, \xi)| \leqq C_{0}^{\prime} \mathrm{e}^{-C_{0}\langle\xi\rangle^{\frac{1}{s}}} .
$$


Since our solution is dependent on the parameter $\varepsilon$ from now on we will adopt the notation $V_{\varepsilon}$. Note that when the initial data are in $\gamma_{c}^{s}\left(\mathbb{R}^{n}\right)$ we do not need any regularisation to embed them in the algebra $\mathcal{G}^{s}\left(\mathbb{R}^{n}\right)$, due to Proposition 4.1(ii). Hence,

$$
\left|V_{\varepsilon}(t, \xi)\right| \leqq C \omega(\varepsilon)^{-2 L}\langle\xi\rangle^{\frac{k}{2 \sigma}}\left|V_{\varepsilon}(0, \xi)\right| \mathrm{e}^{C \omega(\varepsilon)^{-M}\langle\xi\rangle^{\frac{1}{\sigma}}}
$$

and by simple estimates

$$
\begin{aligned}
\left|V_{\varepsilon}(t, \xi)\right| & \leqq C \omega(\varepsilon)^{-2 L}\langle\xi\rangle \frac{k}{2 \sigma} C_{0}^{\prime} \mathrm{e}^{-C_{0}\langle\xi\rangle^{\frac{1}{s}}} \mathrm{e}^{C \omega(\varepsilon)^{-M}\langle\xi\rangle^{\frac{1}{\sigma}}} \\
& =C C_{0}^{\prime} \omega(\varepsilon)^{-2 L}\langle\xi\rangle^{\frac{k}{2 \sigma}} \mathrm{e}^{-\frac{C_{0}}{2}\langle\xi\rangle^{\frac{1}{s}}} \mathrm{e}^{-\frac{C_{0}}{2}\langle\xi\rangle^{\frac{1}{s}}+C \omega(\varepsilon)^{-M}\langle\xi\rangle^{\frac{1}{\sigma}}}
\end{aligned}
$$

If $s<\sigma$, the condition

$$
-\frac{C_{0}}{2}+C \omega(\varepsilon)^{-M}\langle\xi\rangle^{\frac{1}{\sigma}-\frac{1}{s}} \leqq 0
$$

is equivalent to

$$
\begin{aligned}
C \omega(\varepsilon)^{-M}\langle\xi\rangle^{\frac{1}{\sigma}-\frac{1}{s}} & \leqq \frac{C_{0}}{2} \\
\langle\xi\rangle^{\frac{1}{\sigma}-\frac{1}{s}} & \leqq \frac{C_{0}}{2} \frac{1}{C} \omega(\varepsilon)^{M}, \\
\langle\xi\rangle^{\frac{1}{s}-\frac{1}{\sigma}} & \geqq\left(\frac{C_{0}}{2} \frac{1}{C}\right)^{-1} \omega(\varepsilon)^{-M}, \\
\langle\xi\rangle & \geqq\left(\left(\frac{C_{0}}{2} \frac{1}{C}\right)^{-1} \omega(\varepsilon)^{-M}\right)^{\frac{1}{\frac{1}{s}-\frac{1}{\sigma}}}
\end{aligned}
$$

or, in other words, to the condition

$$
\langle\xi\rangle \geqq R_{\varepsilon}:=\left(\left(\frac{C_{0}}{2} \frac{1}{C}\right)^{-1} \omega^{-M}(\varepsilon)\right)^{\frac{1}{\frac{1}{s}-\frac{1}{\sigma}}} .
$$

Assume now that $\omega(\varepsilon)^{-1}$ is moderate, that is $\omega(\varepsilon)^{-1} \leqq c \varepsilon^{-r}$ for some $r \geqq 0$. Hence, there exists $N \in \mathbb{N}_{0}$ such that under the assumption (31) the estimate (30) yields

$$
\left|V_{\varepsilon}(t, \xi)\right| \leqq c^{\prime} \varepsilon^{-N} \mathrm{e}^{-C^{\prime}\langle\xi\rangle^{\frac{1}{s}}}
$$

which proves that the net $U_{\varepsilon}=\mathcal{F}^{-1}\left(V_{\varepsilon} 1_{\langle\xi\rangle \geqq R_{\varepsilon}}\right)$ is $\gamma^{s}$-moderate. It remains to estimate $V_{\varepsilon}(t, \xi)$ when $\langle\xi\rangle \leqq R_{\varepsilon}$. Going back to (30) we have that if $\langle\xi\rangle \leqq R_{\varepsilon}$ then

$$
\begin{aligned}
\left|V_{\varepsilon}(t, \xi)\right| & \leqq C \omega(\varepsilon)^{-2 L}\langle\xi\rangle^{\frac{k}{2 \sigma}} C_{0}^{\prime} \mathrm{e}^{-C_{0}\langle\xi\rangle^{\frac{1}{s}}} \mathrm{e}^{C \omega(\varepsilon)^{-M}\langle\xi\rangle^{\frac{1}{\sigma}}} \\
& \leqq C C_{0}^{\prime} \omega(\varepsilon)^{-2 L}\langle\xi\rangle^{\frac{k}{2 \sigma}} \mathrm{e}^{-\frac{C_{0}}{2}\langle\xi\rangle^{\frac{1}{s}}} \mathrm{e}^{-\frac{C_{0}}{2}\langle\xi\rangle^{\frac{1}{s}}} \mathrm{e}^{C \omega(\varepsilon)^{-M}\left\langle R_{\varepsilon}\right\rangle^{\frac{1}{\sigma}}}
\end{aligned}
$$


At this point, choosing $\omega(\varepsilon)^{-M}\left\langle R_{\varepsilon}\right\rangle^{\frac{1}{\sigma}}$ of logarithmic type, that is,

$$
\begin{aligned}
& \omega(\varepsilon)^{-M}\left\langle R_{\varepsilon}\right\rangle^{\frac{1}{\sigma}} \leqq c \log \left(\varepsilon^{-1}\right) \Longleftrightarrow \omega(\varepsilon)^{-M} \omega(\varepsilon)^{\frac{-M \frac{1}{\sigma}}{\frac{1}{s}-\frac{1}{\sigma}}} \leqq c \log \left(\varepsilon^{-1}\right) \\
& \Longleftrightarrow \omega(\varepsilon)^{-1} \leqq c\left(\log \left(\varepsilon^{-1}\right)\right)^{\frac{1}{M+\frac{M \frac{1}{\sigma}}{\frac{1}{s}-\frac{1}{\sigma}}}} \Longleftrightarrow \omega(\varepsilon)^{-1} \leqq c\left(\log \left(\varepsilon^{-1}\right)\right)^{\frac{\frac{1}{s}-\frac{1}{\sigma}}{\frac{1}{s} M}},
\end{aligned}
$$

we can conclude that there exists $N \in \mathbb{N}_{0}$ and $c^{\prime}, C^{\prime}>0$ such that

$$
\left|V_{\varepsilon}(t, \xi)\right| \leqq c^{\prime} \mathrm{e}^{-C^{\prime}\langle\xi\rangle^{\frac{1}{s}}} \varepsilon^{-N}
$$

for all $\varepsilon \in(0,1], t \in[0, T]$ and $\langle\xi\rangle \leqq R_{\varepsilon}$. This together with (32) and Proposition 4.3(iii) shows that the net $\left(U_{\varepsilon}(t, \cdot)\right)_{\varepsilon}$ is $\gamma^{s}$-moderate on $\mathbb{R}^{n}$ for

$$
1<s<\sigma=1+\frac{k}{2} \text {. }
$$

We are now ready to state and prove the following well-posedness theorem.

\section{Theorem 4.7. Let}

$$
\begin{gathered}
D_{t}^{2} u(t, x)-\sum_{i=1}^{n} b_{i}(t) D_{t} D_{x_{i}} u(t, x)-\sum_{i=1}^{n} a_{i}(t) D_{x_{i}}^{2} u(t, x)=0, \\
u(0, x)=g_{0}, \\
D_{t} u(0, x)=g_{1},
\end{gathered}
$$

where the coefficients $a_{i}$ and $b_{i}$ are real-valued distributions with compact support contained in $[0, T]$ and $a_{i}$ is non-negative for all $i=1, \ldots, n$. Let $g_{0}$ and $g_{1}$ belong to $\gamma_{c}^{s}\left(\mathbb{R}^{n}\right)$ with $s>1$. Then there exists a suitable embedding of the coefficients $a_{i}$ 's and $b_{i}$ 's into $\mathcal{G}([0, T])$ such that he Cauchy problem above has a unique solution $u \in \mathcal{G}\left([0, T] ; \mathcal{G}^{s}\left(\mathbb{R}^{n}\right)\right)$.

Proof. We begin by writing the equation

$$
D_{t}^{2} u(t, x)-\sum_{i=1}^{n} b_{i}(t) D_{t} D_{x_{i}} u(t, x)-\sum_{i=1}^{n} a_{i}(t) D_{x_{i}}^{2} u(t, x)=0
$$

as an equation in $\mathcal{G}\left([0, T] ; \mathcal{G}^{s}\left(\mathbb{R}^{n}\right)\right)$. This means that we replace the coefficients $a_{i}$ and $b_{i}$ with the equivalence classes of $\left(a_{i, \varepsilon}\right)_{\varepsilon}$ and $\left(b_{i, \varepsilon}\right)_{\varepsilon}$ in $\mathcal{G}([0, T])$ as in Section 3. Since the initial data are in $\mathcal{G}^{s}\left(\mathbb{R}^{n}\right)$ they can be imbedded in $\mathcal{G}^{s}\left(\mathbb{R}^{n}\right)$ as they are, that is $\left[\left(g_{0}\right)\right] \in \mathcal{G}_{c}^{s}\left(\mathbb{R}^{n}\right)$ and $\left[\left(g_{1}\right)\right] \in \mathcal{G}_{c}^{s}\left(\mathbb{R}^{n}\right)$.

Existence. We argue now at the level of representatives and we transform the equation to the first order system (6). From the theory of weakly hyperbolic equations and in particular from $[17,23]$ we know that the Cauchy problem

$$
D_{t}^{2} u(t, x)-\sum_{i=1}^{n} b_{i, \varepsilon}(t) D_{t} D_{x_{i}} u(t, x)-\sum_{i=1}^{n} a_{i, \varepsilon}(t) D_{x_{i}}^{2} u(t, x)=0
$$


with initial data $g_{0}, g_{1} \in \gamma_{c}^{s}\left(\mathbb{R}^{n}\right)$, has a net of (classical) solutions $\left(u_{\varepsilon}\right)_{\varepsilon} \in$ $C^{2}\left([0, T]: \gamma^{s}\left(\mathbb{R}^{n}\right)\right)$. More precisely, we know that given $s>1$ and for $k \geqq 2$ there exists a solution $\left(u_{\varepsilon}\right)_{\varepsilon} \in C^{2}\left([0, T]: \gamma^{s}\left(\mathbb{R}^{n}\right)\right)$ provided that

$$
1<s<1+\frac{k}{2}
$$

So, in the arguments which follow we assume $s$ and $k$ in this relation and we perform the embedding of the coefficients $a_{i}$ and $b_{i}$ with a logarithmic scale of the type

$$
\omega^{-1}(\varepsilon)=c\left(\log \left(\varepsilon^{-1}\right)\right)^{r}, \quad c \geqq 0,
$$

as in (33), where $r$ depends on $s$ and $k$.

It is our task to show that this net is moderate. From the energy estimates in Section 4.2 at the Fourier transform level we have that the net $\left(u_{\varepsilon}\right)_{\varepsilon}$ (or better the corresponding $\left.\left(U_{\varepsilon}\right)_{\varepsilon}\right)$ is $\gamma^{s}\left(\mathbb{R}^{n}\right)$-moderate with respect to $x$ with $s$ as above. Since this moderateness estimate is uniform in $t$ and the coefficients of the equation are smooth and moderate in $t \in[0, T]$ as well, by induction on the $t$-derivatives and arguing as in [24] we can easily conclude that $\left(u_{\varepsilon}\right)_{\varepsilon}$ is $C^{\infty}\left([0, T] ; \gamma^{\sigma}\left(\mathbb{R}^{n}\right)\right)$ moderate for

$$
1<s<1+\frac{k}{2}
$$

Hence, $\left(u_{\varepsilon}\right)_{\varepsilon}$ generates a solution $u \in \mathcal{G}\left([0, T] ; \mathcal{G}^{s}\left(\mathbb{R}^{n}\right)\right)$ to our Cauchy problem. Uniqueness. Assume now that the Cauchy problem has another solution $v \in$ $\mathcal{G}\left([0, T] ; \mathcal{G}^{s}\left(\mathbb{R}^{n}\right)\right)$. At the level of representatives this means

$$
\begin{gathered}
D_{t}^{2}\left(u_{\varepsilon}-v_{\varepsilon}\right)(t, x)-\sum_{i=1}^{n} b_{i, \varepsilon}(t) D_{t} D_{x_{i}}\left(u_{\varepsilon}-v_{\varepsilon}\right)(t, x) \\
-\sum_{i=1}^{n} a_{i, \varepsilon}(t) D_{x_{i}}^{2}\left(u_{\varepsilon}-v_{\varepsilon}\right)(t, x)=f_{\varepsilon}(t, x),
\end{gathered}
$$

with initial data

$$
\begin{aligned}
u_{\varepsilon}(0, x)-v_{\varepsilon}(0, x) & =n_{0, \varepsilon}(x), \\
D_{t} u_{\varepsilon}(0, x)-D_{t} v_{\varepsilon}(0, x) & =n_{1, \varepsilon}(x),
\end{aligned}
$$

where $\left(f_{\varepsilon}\right)_{\varepsilon}$ is $C^{\infty}\left([0, T] ; \gamma^{s}\left(\mathbb{R}^{n}\right)\right)$-negligible and $\left(n_{0, \varepsilon}\right)_{\varepsilon}$ and $\left(n_{1, \varepsilon}\right)_{\varepsilon}$ are both compactly supported and $\gamma^{s}\left(\mathbb{R}^{n}\right)$-negligible. The corresponding first order system is

$$
D_{t}\left(\begin{array}{l}
w_{1, \varepsilon} \\
w_{2, \varepsilon}
\end{array}\right)=\left(\begin{array}{cc}
0 & \left\langle D_{x}\right\rangle \\
\sum_{i=1}^{n} a_{i, \varepsilon}(t) D_{x_{i}}^{2}\left\langle D_{x}\right\rangle^{-1} & \sum_{i=1}^{n} b_{i, \varepsilon}(t) D_{x_{i}}
\end{array}\right)\left(\begin{array}{l}
w_{1, \varepsilon} \\
w_{2, \varepsilon}
\end{array}\right)+\left(\begin{array}{c}
0 \\
f_{\varepsilon}
\end{array}\right),
$$

where $w_{1, \varepsilon}$ and $w_{2, \varepsilon}$ are obtained via the transformation

$$
w_{j, \varepsilon}=D_{t}^{j-1}\left\langle D_{x}\right\rangle^{2-j}\left(u_{\varepsilon}-v_{\varepsilon}\right), \quad j=1,2 .
$$


This system will be studied after Fourier transform, as a system of the type

$$
D_{t} V_{\varepsilon}(t, \xi)=\langle\xi\rangle A_{\varepsilon}(t, \xi) V+F_{\varepsilon}
$$

with

$$
F_{\varepsilon}=\left(\begin{array}{c}
0 \\
\mathcal{F}_{x \rightarrow \xi} f_{\varepsilon}
\end{array}\right) .
$$

These kinds of systems and the corresponding weakly hyperbolic equations (with right hand-side) have been investigated in [16] under even less regular assumptions on the coefficients (Hölder). In particular (see Theorem 3 in [16]) Gevrey wellposedness results have been obtained for

$$
1<s<1+\frac{k}{2}
$$

The proof of Theorem 3 in [16] can be easily adapted to our situation by inserting everywhere a multiplicative factor $\omega(\varepsilon)^{-L}$ coming from the regularisation of the coefficients and by replacing $\mathrm{e}^{-\rho(t)\langle\xi\rangle^{\frac{1}{s}}}$ with $\mathrm{e}^{-\rho(t) \varepsilon^{\frac{1}{s}}\langle\xi\rangle^{\frac{1}{s}}}$ in the formula (4.1) defining $V$ in [16]. The estimate (4.9) in [16] is therefore transformed into

$$
\begin{aligned}
\left|V_{\varepsilon}(t, \xi)\right| \leqq & c_{1} \omega(\varepsilon)^{-N}\langle\xi\rangle^{N} \mathrm{e}^{\kappa_{1} \varepsilon^{\frac{1}{s}}\langle\xi\rangle^{\frac{1}{s}}}\left|V_{\varepsilon}(0, \xi)\right| \\
& +c_{2} \omega(\varepsilon)^{-N}\langle\xi\rangle^{N} \mathrm{e}^{\kappa_{2} \varepsilon^{\frac{1}{s}}\langle\xi\rangle^{\frac{1}{s}}}\left|\widehat{F}_{\varepsilon}(t, \xi)\right|,
\end{aligned}
$$

where $N \in \mathbb{N}_{0}$ depends on the equation or better on the regularity of the coefficients and $\kappa_{1}, \kappa_{2}>0$ can be chosen small enough. It follows that since the initial data $V_{\varepsilon}(0, \xi)$ and the right-hand side $F_{\varepsilon}(t, \xi)$ are negligible then $\left(V_{\varepsilon}\right)_{\varepsilon}$ is negligible as well in the suitable function spaces, or in other words, $\left(u_{\varepsilon}-v_{\varepsilon}\right)_{\varepsilon}$ is $C\left([0, T], \gamma^{s}\left(\mathbb{R}^{n}\right)\right)$-negligible. From the equation itself and the fact that the coefficients are nets of smooth functions one can deduce that the net $\left(u_{\varepsilon}-v_{\varepsilon}\right)_{\varepsilon}$ is smooth in $t$ as well and more precisely that it is $C^{\infty}\left([0, T], \gamma^{s}\left(\mathbb{R}^{n}\right)\right)$-negligible. This proves that $u=v$ in $\mathcal{G}\left([0, T] ; \mathcal{G}^{s}\left(\mathbb{R}^{n}\right)\right)$.

\section{Case 2: Well-Posedness for Smooth Initial Data}

We now work under the assumption that the initial data $g_{0}$ and $g_{1}$ are not Gevrey but still smooth. More precisely, $g_{0}, g_{1} \in C_{c}^{\infty}\left(\mathbb{R}^{n}\right)$. By convolution with a mollifier $\varphi_{\varepsilon}$ as in Case 1 we get a net of smooth functions. It is our aim to find for a function $u \in C_{c}^{\infty}\left(\mathbb{R}^{n}\right)$ a new regularisation of the type

$$
u * \rho_{\varepsilon}
$$

such that the corresponding net is Gevrey. This will allow us to embed the initial data $g_{0}$ and $g_{1}$ in an algebra of Gevrey-Colombeau type and to proceed with the well-posedness of the Cauchy problem (3).

We begin with the following regularisation inspired by [1]. 


\subsection{Gevrey Regularisation of Smooth Functions with Compact Support}

In the sequel $\mathcal{S}^{(\sigma)}\left(\mathbb{R}^{n}\right), \sigma>1$, denotes the space of all $\varphi \in \mathcal{C}^{\infty}\left(\mathbb{R}^{n}\right)$ such that

$$
\|\varphi\|_{b, \sigma}=\sup _{\alpha, \beta \in \mathbb{N}_{0}^{n}} \int_{\mathbb{R}^{n}} \frac{\left|x^{\beta}\right|}{b^{|\alpha+\beta|} \alpha !^{\sigma} \beta !^{\sigma}}\left|\partial^{\alpha} \varphi(x)\right| \mathrm{d} x<\infty
$$

for all $b>0$.

We recall that the Gelfand-Shilov space $\mathcal{S}^{(\sigma)}\left(\mathbb{R}^{n}\right)$ is Fourier transform invariant (see for example [27, Chapter 6] and [31]). It follows that taking the inverse Fourier transform $\phi=\mathcal{F}^{-1} \psi$ of a function $\psi \in \mathcal{S}^{(\sigma)}\left(\mathbb{R}^{n}\right)$ identically 1 in a neighborhood of 0 one gets a function $\phi \in \mathcal{S}^{(\sigma)}\left(\mathbb{R}^{n}\right)$ with

$$
\int \phi(x) \mathrm{d} x=1, \quad \text { and } \quad \int x^{\alpha} \phi(x) \mathrm{d} x=0, \quad \text { for all } \alpha \neq 0 .
$$

For instance, one can take $\psi \in \gamma^{(\sigma)}\left(\mathbb{R}^{n}\right) \cap \mathcal{C}_{\mathrm{c}}^{\infty}\left(\mathbb{R}^{n}\right)$, where $\gamma^{(\sigma)}\left(\mathbb{R}^{n}\right)$ is the space of all $f \in \mathcal{C}^{\infty}\left(\mathbb{R}^{n}\right)$ such that for all compact subset $K$ of $\mathbb{R}^{n}$ and all $b>0$ there exists $c>0$ such that

$$
\sup _{x \in K}\left|\partial^{\alpha} f(x)\right| \leqq c b^{|\alpha|} \alpha !^{\sigma}
$$

for all $\alpha \in \mathbb{N}_{0}^{n}$.

We say that $\phi \in \mathcal{S}^{(\sigma)}\left(\mathbb{R}^{n}\right)$ is a mollifier if the property (35) holds. Let now $\chi \in \gamma^{\sigma}\left(\mathbb{R}^{n}\right)$ with $0 \leqq \chi \leqq 1, \chi(x)=0$ for $|x| \geqq 2$ and $\chi(x)=1$ for $|x| \leqq 2$. We define (as in [1]) the net of Gevrey functions

$$
\rho_{\varepsilon}(x):=\varepsilon^{-n} \phi\left(\frac{x}{\varepsilon}\right) \chi(x|\log \varepsilon|) .
$$

Note that the following estimates are valid for $\varepsilon$ small enough, that is, for all $\varepsilon \in(0, \eta]$ with $\eta \in(0,1]$. Without loss of generality we can assume $\eta=1$.

Proposition 5.1. Let $u \in C_{c}^{\infty}\left(\mathbb{R}^{n}\right)$ and $\rho_{\varepsilon}$ as above. Then, there exists $K \Subset \mathbb{R}^{n}$ such that $\operatorname{supp}\left(u * \rho_{\varepsilon}\right) \subseteq K$ for all $\varepsilon$ small enough and

(i) there exists $c>0$ and $\eta \in(0,1]$ such that

$$
\left|\partial^{\alpha}\left(u * \rho_{\varepsilon}\right)(x)\right| \leqq c^{|\alpha|+1}(\alpha !)^{\sigma} \varepsilon^{-|\alpha|}
$$

for all $\alpha \in \mathbb{N}_{0}^{n}, x \in \mathbb{R}^{n}$ and $\varepsilon \in(0, \eta]$, or in other words, $\left(u * \rho_{\varepsilon}\right)_{\varepsilon}$ is $\gamma_{c}^{\sigma}$-moderate.

(ii) The net $\left(u * \rho_{\varepsilon}-u\right)_{\varepsilon}$ is compactly supported uniformly in $\varepsilon$ and $C^{\infty}$-negligible.

(iii) There exist $c, c^{\prime}>0$ and $\eta \in(0,1]$ such that

$$
\left|\widehat{u_{\varepsilon}}(\xi)\right| \leqq c^{\prime} \mathrm{e}^{-c \varepsilon^{\frac{1}{\sigma}}\langle\xi\rangle^{\frac{1}{\sigma}}}
$$

for all $\xi \in \mathbb{R}^{n}$ and $\varepsilon \in(0, \eta]$. 
Proof. (i) We begin by observing that there exists a compact set $K \subseteq \mathbb{R}^{n}$ such that $\operatorname{supp}\left(u * \rho_{\varepsilon}\right) \subseteq K$ for all $\varepsilon \in(0,1 / 2]$. Indeed, since the function $u$ has compact support and $\operatorname{supp} \rho_{\varepsilon} \subseteq|\log \varepsilon|^{-1}(\operatorname{supp} \chi)$ we get the inclusion

$$
\operatorname{supp}\left(u * \rho_{\varepsilon}\right) \subseteq \operatorname{supp} u+|\log (1 / 2)|^{-1} \operatorname{supp} \chi .
$$

We write $\partial^{\alpha}\left(u * \rho_{\varepsilon}\right)(x)$ as

$$
\left(u * \partial^{\alpha} \rho_{\varepsilon}\right)(x)=\varepsilon^{-n} \sum_{\gamma \leqq \alpha}\left(\begin{array}{l}
\alpha \\
\gamma
\end{array}\right) \partial^{\gamma} \phi\left(\frac{x}{\varepsilon}\right) \varepsilon^{-|\gamma|} \partial^{\alpha-\gamma} \chi(x|\log \varepsilon|)|\log \varepsilon|^{|\alpha-\gamma|} .
$$

Hence, the change of variable $y / \varepsilon=z$ in

$$
\int_{\mathbb{R}^{n}} u(x-y) \partial^{\gamma} \phi\left(\frac{y}{\varepsilon}\right) \partial^{\alpha-\gamma} \chi(y|\log \varepsilon|) \mathrm{d} y
$$

entails

$$
\begin{aligned}
& \left|\partial^{\alpha}\left(u * \rho_{\varepsilon}\right)(x)\right| \\
& \quad \leqq \sum_{\gamma \leqq \alpha}\left(\begin{array}{l}
\alpha \\
\gamma
\end{array}\right) \varepsilon^{-|\gamma|}|\log \varepsilon|^{|\alpha-\gamma|} \int_{\mathbb{R}^{n}}|u(x-\varepsilon z)|\left|\partial^{\gamma} \phi(z)\right|\left|\partial^{\alpha-\gamma} \chi(\varepsilon|\log \varepsilon| z)\right| \mathrm{d} z .
\end{aligned}
$$

Since $\chi \in \gamma^{\sigma}\left(\mathbb{R}^{n}\right)$ is compactly supported, there exists a constant $c_{\varphi}>0$ such that

$$
\left|\partial^{\alpha-\gamma} \chi(\varepsilon|\log \varepsilon| z)\right| \leqq c_{\chi}^{|\alpha-\gamma|+1}(\alpha-\gamma) !^{\sigma},
$$

for all $z \in \mathbb{R}^{n}$ and $\varepsilon \in(0,1 / 2]$. Thus, combining (37) with (38) we obtain the estimate

$$
\begin{aligned}
& \left|\partial^{\alpha}\left(u * \rho_{\varepsilon}\right)(x)\right| \leqq \sum_{\gamma \leqq \alpha}\left(\begin{array}{l}
\alpha \\
\gamma
\end{array}\right) \varepsilon^{-|\gamma|}|\log \varepsilon|^{|\alpha-\gamma|} c_{\chi}^{|\alpha-\gamma|+1}(\alpha-\gamma) !^{\sigma} \\
& \quad \times \int_{\mathbb{R}^{n}} \frac{|u(x-\varepsilon z)|\left|\partial^{\gamma} \phi(z)\right|(\gamma !)^{\sigma}}{(\gamma !)^{\sigma}} \mathrm{d} z \\
& \leqq c(u, \chi) \sum_{\gamma \leqq \alpha}\left(\begin{array}{l}
\alpha \\
\gamma
\end{array}\right) \varepsilon^{-|\gamma|}|\log \varepsilon|^{|\alpha-\gamma|} c_{\chi}^{|\alpha-\gamma|}(\alpha-\gamma) !^{\sigma}\|\phi\|_{\sigma, 1} \gamma !^{\sigma} \\
& \leqq c(u, \chi, \phi) \sum_{\gamma \leqq \alpha}\left(\begin{array}{l}
\alpha \\
\gamma
\end{array}\right) \varepsilon^{-|\gamma|}|\log \varepsilon|^{|\alpha-\gamma|} c_{\chi}^{|\alpha-\gamma|}(\alpha-\gamma) !^{\sigma} \gamma !^{\sigma} .
\end{aligned}
$$

Since $|\log \varepsilon|$ is bounded by $\varepsilon^{-1}$,

$$
\sum_{\gamma \leqq \alpha}\left(\begin{array}{l}
\alpha \\
\gamma
\end{array}\right)=2^{|\alpha|}
$$

and

$$
\delta ! \leqq|\delta| ! \leqq|\delta| \delta \mid
$$


for all $\delta \in \mathbb{N}_{0}^{n}$, we can conclude from (39) that

$$
\begin{aligned}
\left|\partial^{\alpha}\left(u * \rho_{\varepsilon}\right)(x)\right| & \leqq c c_{1}^{|\alpha|} \varepsilon^{-|\alpha|} \sum_{\gamma \leqq \alpha}\left(\begin{array}{l}
\alpha \\
\gamma
\end{array}\right)|\alpha-\gamma|^{\sigma|\alpha-\gamma|}|\gamma|^{\sigma|\gamma|} \leqq c c_{1}^{|\alpha|} \varepsilon^{-|\alpha|} 2^{|\alpha|}|\alpha|^{\sigma|\alpha|} \\
& \leqq c^{\prime} c_{1}^{|\alpha|} \varepsilon^{-|\alpha|} 2^{|\alpha|} \mathrm{e}^{\sigma|\alpha|}(\alpha !)^{\sigma}
\end{aligned}
$$

At this point collecting the terms with exponent $|\alpha|$ we conclude that there exist a constants $C>0$ and $C_{1}>0$ such that

$$
\left|\partial^{\alpha}\left(u * \rho_{\varepsilon}\right)(x)\right| \leqq C^{|\alpha|}(\alpha !)^{\sigma} \varepsilon^{-|\alpha|},
$$

uniformly in $\varepsilon \in(0,1 / 2]$.

(ii) By embedding of $C_{c}^{\infty}\left(\mathbb{R}^{n}\right)$ into the Colombeau algebra $\mathcal{G}\left(\mathbb{R}^{n}\right)$ we know that the net $\left(u-u * \phi_{\varepsilon}\right)_{\varepsilon}$ is $C^{\infty}$-negligible. It is easy to check that $\left(u * \phi_{\varepsilon}-u * \rho_{\varepsilon}\right)_{\varepsilon}$ is $C^{\infty}$-negligible as well. Hence, $\left(u-u * \rho_{\varepsilon}\right)_{\varepsilon}$ is $C^{\infty}$-negligible.

(iii) In (i) we have proven that the net $\left(u * \rho_{\varepsilon}\right)_{\varepsilon}$ is $\gamma_{c}^{\sigma}$-moderate and has support contained in a compact set $K$ uniformly with respect to $\varepsilon$. So, by Proposition 4.3(i) we immediately conclude that there exist $c, c^{\prime}>0$ such that

$$
\left|\widehat{u_{\varepsilon}}(\xi)\right| \leqq c^{\prime} \mathrm{e}^{-c \varepsilon^{\frac{1}{\sigma}}\langle\xi\rangle^{\frac{1}{\sigma}}}
$$

for all $\xi \in \mathbb{R}^{n}$ and $\varepsilon \in(0,1 / 2]$.

In the sequel $\iota$ denotes the map

$$
C_{c}^{\infty}\left(\mathbb{R}^{n}\right) \rightarrow \mathcal{G}_{c}^{\sigma}\left(\mathbb{R}^{n}\right): u \mapsto\left[\left(u * \rho_{\varepsilon}\right)_{\varepsilon}\right] .
$$

Proposition 5.2. (i) The map ı is injective on $C_{c}^{\infty}\left(\mathbb{R}^{n}\right)$.

(ii) If $u \in \gamma_{c}^{\sigma}\left(\mathbb{R}^{n}\right)$ then $\left(u * \phi_{\varepsilon}-u * \rho_{\varepsilon}\right)_{\varepsilon}$ is $\gamma^{\sigma}$-negligible.

Proof. (i) Let $u \in C_{c}^{\infty}\left(\mathbb{R}^{n}\right)$ and $\left(u * \rho_{\varepsilon}\right)_{\varepsilon}$ be $\gamma_{c}^{\sigma}$-negligible. Hence, $\left(u * \rho_{\varepsilon}\right)_{\varepsilon}$ is $C^{\infty}$-negligible. Since $\left(u * \phi_{\varepsilon}-u * \rho_{\varepsilon}\right)_{\varepsilon}$ is $C^{\infty}$-negligible we conclude that the net $\left(u * \phi_{\varepsilon}\right)_{\varepsilon}$ is $C^{\infty}$-negligible. By embedding of $C^{\infty}\left(\mathbb{R}^{n}\right)$ into the Colombeau algebra $\mathcal{G}\left(\mathbb{R}^{n}\right)$ it follows that $u=0$. This shows that the map $\iota$ is injective.

(ii) We write $\left(u * \phi_{\varepsilon}-u * \rho_{\varepsilon}\right)(x)$ as

$$
\int_{\mathbb{R}^{n}} u(x-\varepsilon y) \phi(y)(1-\chi(y \varepsilon|\log (\varepsilon)|) \mathrm{d} y .
$$

Hence, by the properties of $\chi$ and by the vanishing moments of $\varphi$, for any integer $q>1$ we get

$$
\begin{aligned}
& \left|\partial^{\alpha}\left(u * \phi_{\varepsilon}-u * \rho_{\varepsilon}\right)(x)\right| \\
& \quad \leqq \int_{\mathbb{R}^{n}}\left|\partial^{\alpha} u(x-\varepsilon y)\right||\phi(y)| \sum_{|\beta|=q} \frac{\left|\partial^{\beta} \chi(y \varepsilon|\log \varepsilon| \theta)\right|}{\beta !}|\varepsilon \log (\varepsilon) y|^{|\beta|} \mathrm{d} y \\
& \quad \leqq c^{|\alpha|+1}(\alpha !)^{\sigma} \int_{\mathbb{R}^{n}}|\phi(y)||y|^{q} c(\chi, q)|\varepsilon \log (\varepsilon)|^{q} \mathrm{~d} y \\
& \quad \leqq c(q, \chi, \phi) c^{|\alpha|+1}(\alpha !)^{\sigma} \varepsilon^{\frac{q}{2}} .
\end{aligned}
$$

This proves that the net $\left(u * \phi_{\varepsilon}-u * \rho_{\varepsilon}\right)(x)$ is $\gamma^{\sigma}$-negligible. 
Concluding, we can state that the algebra $\mathcal{G}_{c}^{\sigma}\left(\mathbb{R}^{n}\right)$ contains not only $\gamma_{c}^{\sigma}\left(\mathbb{R}^{n}\right)$ but also $C_{c}^{\infty}\left(\mathbb{R}^{n}\right)$ as a subalgebra. This is obtained by modifying the embedding from $u * \varphi_{\varepsilon}$ in Section 4 to $u * \rho_{\varepsilon}$.

\subsection{Energy Estimates and Well-Posedness}

We now take initial data $g_{0}, g_{1}$ in $C_{c}^{\infty}\left(\mathbb{R}^{n}\right)$ and we embed them in $\mathcal{G}_{c}^{s}\left(\mathbb{R}^{n}\right)$ as $g_{0} * \rho_{\varepsilon}$ and $g_{1} * \rho_{\varepsilon}$. By repeating the transformation into first order system and the energy estimates of Case 1 at the Fourier transform level we arrive at (29), that is

$$
\left|V_{\varepsilon}(t, \xi)\right| \leqq C \omega(\varepsilon)^{-2 L}\langle\xi\rangle^{\frac{k}{2 \sigma}}\left|V_{\varepsilon}(0, \xi)\right| \mathrm{e}^{C \omega(\varepsilon)^{-M}\langle\xi\rangle^{\frac{1}{\sigma}}}
$$

for a suitable constant $C>0$ and $M=(3 L+k) / k$. Since

$$
\left|V_{\varepsilon}(0, \xi)\right| \leqq C_{0}^{\prime} \mathrm{e}^{-C_{0} \varepsilon^{\frac{1}{s}}\langle\xi\rangle^{\frac{1}{s}}}
$$

we get

$$
\begin{aligned}
\left|V_{\varepsilon}(t, \xi)\right| & \leqq C \omega(\varepsilon)^{-2 L}\langle\xi\rangle^{\frac{k}{2 \sigma}} C_{0}^{\prime} \mathrm{e}^{-C_{0} \varepsilon^{\frac{1}{s}}\langle\xi\rangle^{\frac{1}{s}}} \mathrm{e}^{C \omega(\varepsilon)^{-M}\langle\xi\rangle^{\frac{1}{\sigma}}} \\
& =C C_{0}^{\prime} \omega(\varepsilon)^{-2 L}\langle\xi\rangle^{\frac{k}{2 \sigma}} \mathrm{e}^{-\frac{C_{0}}{2} \varepsilon^{\frac{1}{s}}\langle\xi\rangle^{\frac{1}{s}}} \mathrm{e}^{-\frac{C_{0}}{2} \varepsilon^{\frac{1}{s}}\langle\xi\rangle^{\frac{1}{s}}+C \omega(\varepsilon)^{-M}\langle\xi\rangle^{\frac{1}{\sigma}}}
\end{aligned}
$$

Recall that $s>1$ and that $k$ is any fixed integer with $k \geqq 2$. Now, if $s<\sigma$, the following inequalities are equivalent:

$$
\begin{aligned}
-\frac{C_{0}}{2} \varepsilon^{\frac{1}{s}}+C \omega(\varepsilon)^{-M}\langle\xi\rangle^{\frac{1}{\sigma}-\frac{1}{s}} & \leqq 0, \\
C \omega(\varepsilon)^{-M}\langle\xi\rangle^{\frac{1}{\sigma}-\frac{1}{s}} & \leqq \frac{C_{0}}{2} \varepsilon^{\frac{1}{s}}, \\
\langle\xi\rangle^{\frac{1}{\sigma}-\frac{1}{s}} & \leqq \frac{C_{0}}{2} \frac{1}{C} \omega(\varepsilon)^{M} \varepsilon^{\frac{1}{s}}, \\
\langle\xi\rangle^{\frac{1}{s}-\frac{1}{\sigma}} & \geqq\left(\left(\frac{C_{0}}{2} \frac{1}{C}\right)^{-1} \omega(\varepsilon)^{-M_{\varepsilon}} \varepsilon^{-\frac{1}{s}}\right), \\
\langle\xi\rangle & \geqq\left(\left(\frac{C_{0}}{2} \frac{1}{C}\right)^{-1} \omega(\varepsilon)^{-M_{\varepsilon}-\frac{1}{s}}\right)^{\frac{1}{s}-\frac{1}{\sigma}},
\end{aligned}
$$

or, in other words,

$$
\langle\xi\rangle \geqq R_{\varepsilon}:=\left(\left(\frac{C_{0}}{2} \frac{1}{C}\right)^{-1} \omega(\varepsilon)^{-M_{\varepsilon}} \varepsilon^{-\frac{1}{s}}\right)^{\frac{1}{s}-\frac{1}{\sigma}} .
$$

As in the previous case we take $\omega(\varepsilon)^{-1}$ moderate. Under the assumption (42) the estimate (41) implies, for some $N \in \mathbb{N}_{0}$,

$$
\left|V_{\varepsilon}(t, \xi)\right| \leqq C^{\prime} \varepsilon^{-N} \mathrm{e}^{-C^{\prime} \varepsilon^{\frac{1}{s}}\langle\xi\rangle^{\frac{1}{s}}} .
$$


This shows that the net $U_{\varepsilon}=\mathcal{F}^{-1}\left(V_{\varepsilon} 1_{\langle\xi\rangle \geqq R_{\varepsilon}}\right)$ is $\gamma^{s}$-moderate. We still have to estimate $V_{\varepsilon}(t, \xi)$ when $\langle\xi\rangle \leqq R_{\varepsilon}$. Going back to (41) we have that if $\langle\xi\rangle \leqq R_{\varepsilon}$ then

$$
\begin{aligned}
\left|V_{\varepsilon}(t, \xi)\right| & \leqq C \omega(\varepsilon)^{-2 L}\langle\xi\rangle^{\frac{k}{2 \sigma}} C_{0}^{\prime} \mathrm{e}^{-C_{0} \varepsilon^{\frac{1}{s}}\langle\xi\rangle^{\frac{1}{s}}} \mathrm{e}^{C \omega(\varepsilon)^{-M}\langle\xi\rangle^{\frac{1}{\sigma}}} \\
& \leqq C C_{0}^{\prime} \omega(\varepsilon)^{-2 L}\langle\xi\rangle^{\frac{k}{2 \sigma}} \mathrm{e}^{-\frac{C_{0}}{2} \varepsilon^{\frac{1}{s}}\langle\xi\rangle^{\frac{1}{s}}} \mathrm{e}^{-\frac{C_{0}}{2} \varepsilon^{\frac{1}{s}}\langle\xi\rangle^{\frac{1}{s}}}+C \omega(\varepsilon)^{-M}\left\langle R_{\varepsilon}\right\rangle^{\frac{1}{\sigma}}
\end{aligned}
$$

At this point, choosing $\omega^{-M}(\varepsilon)\left\langle R_{\varepsilon}\right\rangle^{\frac{1}{\sigma}}$ of logarithmic type, that is,

$$
\begin{gathered}
\omega^{-M}(\varepsilon)\left\langle R_{\varepsilon}\right\rangle^{\frac{1}{\sigma}} \leqq c \log \left(\varepsilon^{-1}\right) \Leftrightarrow \omega(\varepsilon)^{-M} \omega(\varepsilon)^{\frac{-M \frac{1}{\sigma}}{\frac{1}{s}-\frac{1}{\sigma}}} \varepsilon^{-\frac{1}{s} \frac{\frac{1}{\sigma}}{\frac{1}{s}-\frac{1}{\sigma}} \leqq c \log \left(\varepsilon^{-1}\right)} \\
\Leftrightarrow \omega(\varepsilon)^{-1} \leqq c\left(\log \left(\varepsilon^{-1}\right)\right)^{\frac{1}{M+\frac{M}{\frac{1}{\sigma}}}} \frac{1 \frac{1}{s}-\frac{1}{\sigma}}{\varepsilon^{\frac{1}{\sigma M}}}, \Leftrightarrow \omega(\varepsilon)^{-1} \leqq c\left(\log \left(\varepsilon^{-1}\right)\right)^{\frac{\frac{1}{s}-\frac{1}{\sigma}}{\frac{1}{\sigma} M}} \varepsilon^{\frac{1}{\sigma M}},
\end{gathered}
$$

we can conclude that there exists $N \in \mathbb{N}_{0}$ and $c^{\prime}, C^{\prime}>0$ such that

$$
\left|V_{\varepsilon}(t, \xi)\right| \leqq c^{\prime} \mathrm{e}^{-C^{\prime} \varepsilon^{\frac{1}{s}}\langle\xi\rangle^{\frac{1}{s}}} \varepsilon^{-N},
$$

for all $\varepsilon \in(0,1], t \in[0, T]$ and $\langle\xi\rangle \leqq R_{\varepsilon}$. Combing this last estimate with (43) we can conclude, by Proposition 4.3(iii), that, as in the previous case, the net $\left(U_{\varepsilon}(t, \cdot)\right)_{\varepsilon}$ is $\gamma^{s}$-moderate on $\mathbb{R}^{n}$ for

$$
1<s<\sigma=1+\frac{k}{2}
$$

We are now ready to state the following well-posedness theorem.

\section{Theorem 5.3. Let}

$$
\begin{gathered}
D_{t}^{2} u(t, x)-\sum_{i=1}^{n} b_{i}(t) D_{t} D_{x_{i}} u(t, x)-\sum_{i=1}^{n} a_{i}(t) D_{x_{i}}^{2} u(t, x)=0, \\
u(0, x)=g_{0}, \\
D_{t} u(0, x)=g_{1},
\end{gathered}
$$

where the coefficients $a_{i}$ and $b_{i}$ are real valued distributions with compact support contained in $[0, T]$ and $a_{i}$ is non-negative for all $i=1, \ldots, n$. Let $g_{0}$ and $g_{1}$ belong to $C_{c}^{\infty}\left(\mathbb{R}^{n}\right)$. Then, for all $s>1$ there exists a suitable embedding of the coefficients $a_{i}$ and $b_{i}$ into $\mathcal{G}([0, T])$ such that the Cauchy problem above has a unique solution $u \in \mathcal{G}\left([0, T] ; \mathcal{G}^{s}\left(\mathbb{R}^{n}\right)\right)$.

Proof. We reduce the Cauchy problem above to a first order system and embed of the coefficients and initial data in the corresponding Colombeau algebras $\left(a_{i, \varepsilon} *\right.$ $\left.\psi_{\omega(\varepsilon)}, b_{i, \varepsilon} * \psi_{\omega(\varepsilon)}, g_{0} * \rho_{\varepsilon}, g_{1} * \rho_{\varepsilon}\right)$. Note that, we embed the coefficients $a_{i}$ and $b_{i}$ by means of a net $\omega(\varepsilon)$ with

$$
\omega^{-1}(\varepsilon) \leqq c \varepsilon^{r_{1}}\left(\log \left(\varepsilon^{-1}\right)\right)^{r_{2}}
$$


as in (45), where $r_{1}$ and $r_{2}$ depend on $s$ and fixed $k \geqq 2$ with $1 \leqq s<1+k$.

The energy estimates of Section 5.2 and the same arguments of Case 1 show the existence of a solution $u \in \mathcal{G}\left([0, T] ; \mathcal{G}^{s}\left(\mathbb{R}^{n}\right)\right)$. The uniqueness of the solution $u$ is obtained as in the proof of Theorem 4.7 .

\section{Case 3: Well-Posedness for Distributional Initial Data}

We now consider distributional initial data, that is $g_{0}, g_{1} \in \mathcal{E}^{\prime}\left(\mathbb{R}^{n}\right)$, and investigate their convolution with the mollifier $\rho_{\varepsilon}$.

Proposition 6.1. Let $u \in \mathcal{E}^{\prime}\left(\mathbb{R}^{n}\right)$ and $\rho_{\varepsilon}$ as in (36). Then, there exists $K \Subset \mathbb{R}^{n}$ such that $\operatorname{supp}\left(u * \rho_{\varepsilon}\right) \subseteq K$ for all $\varepsilon$ small enough and there exist $C>0, N \in \mathbb{N}_{0}$ and $\eta \in(0,1]$ such that

$$
\left|\partial^{\alpha}\left(u * \rho_{\varepsilon}\right)(x)\right| \leqq C^{|\alpha|+1}(\alpha !)^{\sigma} \varepsilon^{-|\alpha|-N}
$$

for all $\alpha \in \mathbb{N}_{0}^{n}, x \in \mathbb{R}^{n}$ and $\varepsilon \in(0, \eta]$.

Proof. The following proof differs from the proofs of Propositions 4.1 and 5.1 in terms of mollifier and dependence in $\varepsilon$. We begin by noting that there exists a compact set $K \subseteq \mathbb{R}^{n}$ such that $\operatorname{supp}\left(u * \rho_{\varepsilon}\right) \subseteq K$ for all $\varepsilon \in(0,1 / 2]$. Indeed, since the distribution $u$ has compact support and $\operatorname{supp} \rho_{\varepsilon} \subseteq|\log \varepsilon|^{-1}(\operatorname{supp} \chi)$ we get the inclusion

$$
\operatorname{supp}\left(u * \rho_{\varepsilon}\right) \subseteq \operatorname{supp} u+|\log (1 / 2)|^{-1} \operatorname{supp} \chi
$$

By the structure of $u$ we know that there exists a continuous and compactly supported function $g$ such that

$$
\partial^{\alpha}\left(u * \rho_{\varepsilon}\right)=\partial^{\alpha}\left(\partial^{\beta} g * \rho_{\varepsilon}\right)=g * \partial^{\alpha+\beta} \rho_{\varepsilon},
$$

where

$$
\partial^{\alpha+\beta} \rho_{\varepsilon}=\varepsilon^{-n} \sum_{\gamma \leqq \alpha+\beta}\left(\begin{array}{c}
\alpha+\beta \\
\gamma
\end{array}\right) \partial^{\gamma} \phi\left(\frac{x}{\varepsilon}\right) \varepsilon^{-|\gamma|} \partial^{\alpha+\beta-\gamma} \chi(x|\log \varepsilon|)|\log \varepsilon|^{|\alpha+\beta+\gamma|} .
$$

Hence, the change of variable $y / \varepsilon=z$ in

$$
\int_{\mathbb{R}^{n}} g(x-y) \partial^{\gamma} \phi\left(\frac{y}{\varepsilon}\right) \partial^{\alpha+\beta-\gamma} \chi(y|\log \varepsilon|) \mathrm{d} y
$$

entails

$$
\begin{aligned}
\left|\partial^{\alpha}\left(u * \rho_{\varepsilon}\right)(x)\right| \leqq & \sum_{\gamma \leqq \alpha+\beta}\left(\begin{array}{c}
\alpha+\beta \\
\gamma
\end{array}\right) \varepsilon^{-|\gamma|}|\log \varepsilon|^{|\alpha+\beta+\gamma|} \\
& \times \int_{\mathbb{R}^{n}}|g(x-\varepsilon z)|\left|\partial^{\gamma} \phi(z)\right|\left|\partial^{\alpha+\beta-\gamma} \chi(\varepsilon|\log \varepsilon| z)\right| \mathrm{d} z
\end{aligned}
$$


Since $\chi \in \gamma^{\sigma}\left(\mathbb{R}^{n}\right)$ is compactly supported, there exists a constant $c_{\chi}>0$ such that

$$
\left|\partial^{\alpha+\beta-\gamma} \chi(\varepsilon|\log \varepsilon| z)\right| \leqq c_{\varphi}^{|\alpha+\beta-\gamma|+1}(\alpha+\beta-\gamma) !^{\sigma},
$$

for all $z \in \mathbb{R}^{n}$ and $\varepsilon \in(0,1 / 2]$. Hence, combining (46) with (47) we obtain the estimate

$$
\begin{aligned}
& \left|\partial^{\alpha}\left(u * \rho_{\varepsilon}\right)(x)\right| \leqq \sum_{\gamma \leqq \alpha+\beta}\left(\begin{array}{c}
\alpha+\beta \\
\gamma
\end{array}\right) \varepsilon^{-|\gamma|}|\log \varepsilon|^{|\alpha+\beta+\gamma|} c_{\chi}^{|\alpha+\beta-\gamma|+1}(\alpha+\beta-\gamma) !^{\sigma} \\
& \quad \times \int_{\mathbb{R}^{n}} \frac{|g(x-\varepsilon z)|\left|\partial^{\gamma} \phi(z)\right|(\gamma !)^{\sigma}}{(\gamma !)^{\sigma}} \mathrm{d} z \\
& \leqq c(g, \chi) \sum_{\gamma \leqq \alpha+\beta}\left(\begin{array}{c}
\alpha+\beta \\
\gamma
\end{array}\right) \varepsilon^{-|\gamma|}|\log \varepsilon|^{|\alpha+\beta+\gamma|} c_{\chi}^{|\alpha+\beta-\gamma|}(\alpha+\beta-\gamma) !^{\sigma}\|\phi\|_{\sigma, 1} \gamma !^{\sigma} \\
& \leqq c(g, \chi, \phi) \sum_{\gamma \leqq \alpha+\beta}\left(\begin{array}{c}
\alpha+\beta \\
\gamma
\end{array}\right) \varepsilon^{-|\gamma|}|\log \varepsilon|^{|\alpha+\beta+\gamma|} c_{\chi}^{|\alpha+\beta-\gamma|}(\alpha+\beta-\gamma) !^{\sigma} \gamma !^{\sigma} .
\end{aligned}
$$

Since $|\log \varepsilon|$ is bounded by $\varepsilon^{-1}, \sum_{\gamma} \leqq \alpha+\beta\left(\begin{array}{c}\alpha+\beta \\ \gamma\end{array}\right)=2^{|\alpha+\beta|}$ and $\delta ! \leqq|\delta| ! \leqq|\delta|^{|\delta|}$ for all $\delta \in \mathbb{N}_{0}^{n}$ we can conclude from (48) that

$$
\begin{aligned}
\left|\partial^{\alpha}\left(u * \rho_{\varepsilon}\right)(x)\right| & \leqq c c_{1}^{|\alpha+\beta|} \varepsilon^{-|\alpha|-|\beta|} \sum_{\gamma \leqq \alpha+\beta}\left(\begin{array}{c}
\alpha+\beta \\
\gamma
\end{array}\right)|\alpha+\beta-\gamma|^{\sigma|\alpha+\beta-\gamma|}|\gamma|^{\sigma|\gamma|} \\
& \leqq c c_{1}^{|\alpha+\beta|} \varepsilon^{-|\alpha|-|\beta|} 2^{|\alpha+\beta|}|\alpha+\beta|^{\sigma|\alpha+\beta|} \\
& \leqq c c_{1}^{|\alpha+\beta|} \varepsilon^{-|\alpha|-|\beta|} 2^{|\alpha+\beta|} \mathrm{e}^{\sigma|\alpha+\beta|}|\alpha+\beta| !^{\sigma} .
\end{aligned}
$$

At this point collecting the terms with exponent $|\alpha|$ and the terms with exponent $|\beta|$ ( $\beta$ depends only on $u$ ) we conclude that there exist a constants $C>0$ and $C_{1}>0$ such that

$$
\left|\partial^{\alpha}\left(u * \rho_{\varepsilon}\right)(x)\right| \leqq C_{1}^{|\beta|}|\beta| !^{\sigma} C^{|\alpha|}|\alpha !|^{\sigma}{ }_{\varepsilon}-|\alpha|-|\beta|,
$$

uniformly in $\varepsilon \in(0,1 / 2]$. Note that by the inequality $|\delta| ! \leqq n^{|\delta|} \delta !$ we have that (49) implies the assertion of Proposition 6.1 with $N=|\beta|$ and different constants.

It follows that the net $\left(u * \rho_{\varepsilon}\right)_{\varepsilon}$ is $\gamma_{c}^{\sigma}$-moderate and therefore from Proposition 4.3 we have that there exists $c>0$ and $N \in \mathbb{N}_{0}$ such that

$$
\left|\widehat{u * \rho_{\varepsilon}}(\xi)\right| \leqq c \varepsilon^{-N} \mathrm{e}^{-c \varepsilon^{\frac{1}{\sigma}}\langle\xi\rangle^{\frac{1}{\sigma}}},
$$

for all $\xi \in \mathbb{R}^{n}$ and $\varepsilon$ small enough (from the proof, $\varepsilon \in(0,1 / 2]$ ).

Remark 6.2. Starting from Proposition 6.1 and arguing as for the embedding of $\mathcal{E}^{\prime}\left(\mathbb{R}^{n}\right)$ into $\mathcal{G}\left(\mathbb{R}^{n}\right)$ one can easily prove that

$$
\mathcal{E}^{\prime}\left(\mathbb{R}^{n}\right) \rightarrow \mathcal{G}_{c}^{\sigma}\left(\mathbb{R}^{n}\right): u \mapsto\left[\left(u * \rho_{\varepsilon}\right)_{\varepsilon}\right]
$$

is an embedding of $\mathcal{E}^{\prime}\left(\mathbb{R}^{n}\right)$ into $\mathcal{G}_{c}^{\sigma}\left(\mathbb{R}^{n}\right)$. 


\subsection{Energy Estimates and Well-Posedness}

Let us now consider the Cauchy problem

$$
\begin{gathered}
D_{t}^{2} u(t, x)-\sum_{i=1}^{n} b_{i}(t) D_{t} D_{x_{i}} u(t, x)-\sum_{i=1}^{n} a_{i}(t) D_{x_{i}}^{2} u(t, x)=0, \\
u(0, x)=g_{0}, \\
D_{t} u(0, x)=g_{1},
\end{gathered}
$$

with $g_{0}, g_{1} \in \mathcal{E}^{\prime}\left(\mathbb{R}^{n}\right)$. We embed coefficients and initial data in the corresponding Colombeau algebras and we transform the equation into a first order system similarly to Case 1 and 2. In particular, from Proposition 6.1, we have in this case that the initial data $V_{\varepsilon}(0, \xi)$ fulfils

$$
\left|V_{\varepsilon}(0, \xi)\right| \leqq \varepsilon^{-N} C_{0}^{\prime} \mathrm{e}^{-C_{0} \varepsilon^{\frac{1}{s}}\langle\xi\rangle^{\frac{1}{s}}},
$$

for some $N \in \mathbb{N}_{0}$. This modifies the estimates of Case 2 only by a multiplying factor $\varepsilon^{-N}$, so for $R_{\varepsilon}$ as in (42) we get that there exists $N^{\prime} \in \mathbb{N}_{0}$ such that

$$
\left|V_{\varepsilon}(t, \xi)\right| \leqq c^{\prime} \mathrm{e}^{-C^{\prime} \varepsilon^{\frac{1}{s}}\langle\xi\rangle^{\frac{1}{s}}} \varepsilon^{-N^{\prime}}
$$

for all $\varepsilon, t \in(0, T]$ and $\xi \in \mathbb{R}^{n}$. This result allows us to state the following well-posedness theorem.

Theorem 6.3. Let

$$
\begin{gathered}
D_{t}^{2} u(t, x)-\sum_{i=1}^{n} b_{i}(t) D_{t} D_{x_{i}} u(t, x)-\sum_{i=1}^{n} a_{i}(t) D_{x_{i}}^{2} u(t, x)=0, \\
u(0, x)=g_{0}, \\
D_{t} u(0, x)=g_{1},
\end{gathered}
$$

where the coefficients $a_{i}$ and $b_{i}$ are real valued distributions with compact support contained in $[0, T]$ and $a_{i}$ is non-negative for all $i=1, \ldots, n$. Then, the conclusion of Theorem 5.3 holds for initial data $g_{0}$ and $g_{1}$ in $\mathcal{E}^{\prime}\left(\mathbb{R}^{n}\right)$ as well.

\section{Consistency with the Classical Well-Posedness Results}

We conclude this paper by showing that when the coefficients are regular enough and the initial data are Gevrey then the very weak solution coincides with the classical and ultradistributional ones obtained in [17,23].

Theorem 7.1. Let

$$
\begin{gathered}
D_{t}^{2} u(t, x)-\sum_{i=1}^{n} b_{i}(t) D_{t} D_{x_{i}} u(t, x)-\sum_{i=1}^{n} a_{i}(t) D_{x_{i}}^{2} u(t, x)=0, \\
u(0, x)=g_{0}, \\
D_{t} u(0, x)=g_{1},
\end{gathered}
$$


where the real-valued coefficients $a_{i}$ and $b_{i}$ are compactly supported, belong to $C^{k}([0, T])$ with $k \geqq 2$ and $a_{i} \geqq 0$ for all $i=1, \ldots, n$. Let $g_{0}$ and $g_{1}$ belong to $\gamma_{c}^{s}\left(\mathbb{R}^{n}\right)$ with $s>1$. Then

(i) there exists an embedding of the coefficients $a_{i}$ 's and $b_{i}$ 's, $i=1, \ldots, n$, into $\mathcal{G}([0, T])$, such that the Cauchy problem above has a unique solution $u \in \mathcal{G}\left([0, T] ; \mathcal{G}^{s}\left(\mathbb{R}^{n}\right)\right)$ provided that

$$
1<s<1+\frac{k}{2}
$$

(ii) any representative $\left(u_{\varepsilon}\right)_{\varepsilon}$ of $u$ converges in $C\left([0, T] ; \gamma^{s}\left(\mathbb{R}^{n}\right)\right)$ as $\varepsilon \rightarrow 0$ to the unique classical solution in $C^{2}\left([0, T], \gamma^{s}\left(\mathbb{R}^{n}\right)\right)$ of the Cauchy problem (50);

(iii) if the initial data $g_{0}$ and $g_{1}$ belong to $\mathcal{E}^{\prime}\left(\mathbb{R}^{n}\right)$ then any representative $\left(u_{\varepsilon}\right)_{\varepsilon}$ of $u$ converges in $C\left([0, T] ; \mathcal{D}_{(s)}^{\prime}\left(\mathbb{R}^{n}\right)\right)$ to the ultradistributional solution in $C^{2}\left([0, T], \mathcal{D}_{(s)}^{\prime}\left(\mathbb{R}^{n}\right)\right)$ of the Cauchy problem $(50)$.

Proof. (i) From Section 4 (Case 1) we know that by embedding coefficients and initial data in the corresponding Colombeau algebras the Cauchy problem has a unique solution $u \in \mathcal{G}\left([0, T] ; \mathcal{G}^{s}\left(\mathbb{R}^{n}\right)\right)$. It also has a unique classical solution $\widetilde{u} \in C^{2}\left([0, T], \gamma^{s}\left(\mathbb{R}^{n}\right)\right)$.

(ii) We now want to compare $u$ with $\tilde{u}$. By definition of the classical solution we know that

$$
\begin{gathered}
D_{t}^{2} \widetilde{u}(t, x)-\sum_{i=1}^{n} b_{i}(t) D_{t} D_{x_{i}} \widetilde{u}(t, x)-\sum_{i=1}^{n} a_{i}(t) D_{x_{i}}^{2} \widetilde{u}(t, x)=0, \\
\widetilde{u}(0, x)=g_{0}, \\
D_{t} \widetilde{u}(0, x)=g_{1} .
\end{gathered}
$$

Since the initial data do not need to be regularised because they are already Gevrey, there exists a representative $\left(u_{\varepsilon}\right)_{\varepsilon}$ of $u$ such that

$$
\begin{gathered}
D_{t}^{2} u_{\varepsilon}(t, x)-\sum_{i=1}^{n} b_{i, \varepsilon}(t) D_{t} D_{x_{i}} u_{\varepsilon}(t, x)-\sum_{i=1}^{n} a_{i, \varepsilon}(t) D_{x_{i}}^{2} u_{\varepsilon}(t, x)=0, \\
u_{\varepsilon}(0, x)=g_{0}, \\
D_{t} u_{\varepsilon}(0, x)=g_{1},
\end{gathered}
$$

for suitable embeddings of the coefficients $a_{i}$ and $b_{i}$. Noting that the nets $\left(a_{i, \varepsilon}-a_{i}\right)_{\varepsilon}$ and $\left(b_{i, \varepsilon}-b_{i}\right)_{\varepsilon}$ are converging to 0 in $C\left([0, T] \times \mathbb{R}^{n}\right)$ for $i=1, \ldots, n$ we can rewrite (51) as

$$
\begin{gathered}
D_{t}^{2} \widetilde{u}(t, x)-\sum_{i=1}^{n} b_{i, \varepsilon}(t) D_{t} D_{x_{i}} \widetilde{u}(t, x)-\sum_{i=1}^{n} a_{i, \varepsilon}(t) D_{x_{i}}^{2} \widetilde{u}(t, x)=n_{\varepsilon}(t, x), \\
\widetilde{u}(0, x)=g_{0}, \\
D_{t} \widetilde{u}(0, x)=g_{1},
\end{gathered}
$$


where $n_{\varepsilon} \in C\left([0, T] ; \gamma^{s}\left(\mathbb{R}^{n}\right)\right)$ and converges to 0 in this space. From (53) and (52) we get that $\tilde{u}-u_{\varepsilon}$ solves the Cauchy problem

$$
\begin{gathered}
D_{t}^{2}\left(\widetilde{u}-u_{\varepsilon}\right)(t, x)-\sum_{i=1}^{n} b_{i, \varepsilon}(t) D_{t} D_{x_{i}}\left(\widetilde{u}-u_{\varepsilon}\right)(t, x)-\sum_{i=1}^{n} a_{i, \varepsilon}(t) D_{x_{i}}^{2}\left(\widetilde{u}-u_{\varepsilon}\right)(t, x)=n_{\varepsilon}(t, x), \\
\left(\widetilde{u}-u_{\varepsilon}\right)(0, x)=0, \\
\left(D_{t} \widetilde{u}-D_{t} u_{\varepsilon}\right)(0, x)=0 .
\end{gathered}
$$

By the energy estimates of Case 1 and arguing as in the uniqueness proof of Theorem 4.7 to deal with the right-hand side we arrive after reduction to a system and by application of the Fourier transform to estimate $\left|\left(\widetilde{V}-V_{\varepsilon}\right)(t, \xi)\right|$ as in (34), in terms of $\left(\widetilde{V}-V_{\varepsilon}\right)(0, \xi)$ and the right-hand side $n_{\varepsilon}(t, x)$. In particular, since the coefficients are regular enough (of class $C^{k}, k \geqq 2$ ), the term $\omega(\varepsilon)^{-N}$ disappears in (34) and we simply get

$$
\left|\left(\widetilde{V}-V_{\varepsilon}\right)(t, \xi)\right| \leqq c_{1}\langle\xi\rangle^{N} \mathrm{e}^{\kappa_{1} \varepsilon^{\frac{1}{s}}\langle\xi\rangle^{\frac{1}{s}}}\left|\left(\widetilde{V}-V_{\varepsilon}\right)(0, \xi)\right|+c_{2}\langle\xi\rangle^{N} \mathrm{e}^{\kappa_{2} \varepsilon^{\frac{1}{s}}\langle\xi\rangle^{\frac{1}{s}}}\left|\widehat{n_{\varepsilon}}(t, \xi)\right| .
$$

Since $\left(\widetilde{V}-V_{\varepsilon}\right)(0, \xi)=0$ and $n_{\varepsilon} \rightarrow 0$ in $C\left([0, T] ; \gamma^{s}\left(\mathbb{R}^{n}\right)\right)$ we conclude that $u_{\varepsilon} \rightarrow \widetilde{u}$ in $C\left([0, T] ; \gamma^{s}\left(\mathbb{R}^{n}\right)\right)$. Moreover, since any other representative of $u$ will differ from $\left(u_{\varepsilon}\right)_{\varepsilon}$ by a $C^{\infty}\left([0, T] ; \gamma^{s}\left(\mathbb{R}^{n}\right)\right)$-negligible net, the limit is the same for any representative of $u$.

(iii) Let us now consider the case of initial data in $\mathcal{E}^{\prime}\left(\mathbb{R}^{n}\right)$. We know from [17] that the Cauchy problem

$$
\begin{gathered}
D_{t}^{2} u(t, x)-\sum_{i=1}^{n} b_{i}(t) D_{t} D_{x_{i}} u(t, x)-\sum_{i=1}^{n} a_{i}(t) D_{x_{i}}^{2} u(t, x)=0, \\
u(0, x)=g_{0}, \\
D_{t} u(0, x)=g_{1},
\end{gathered}
$$

has a unique solution $\tilde{u} \in C^{2}\left([0, T], \mathcal{D}_{(s)}^{\prime}\left(\mathbb{R}^{n}\right)\right)$ in the sense of ultradistributions. Hence,

$$
\begin{gathered}
D_{t}^{2} \widetilde{u}(t, x)-\sum_{i=1}^{n} b_{i}(t) D_{t} D_{x_{i}} \widetilde{u}(t, x)-\sum_{i=1}^{n} a_{i}(t) D_{x_{i}}^{2} \widetilde{u}(t, x)=0, \\
\widetilde{u}(0, x)=g_{0}, \\
D_{t} \widetilde{u}(0, x)=g_{1} .
\end{gathered}
$$

We also know that the Cauchy problem (55) has a unique solution $u$ in $\mathcal{G}\left([0, T] ; \mathcal{G}^{s}\right.$ $\left.\left(\mathbb{R}^{n}\right)\right)$ after suitable embedding of coefficients and initial data. This means that there exists a representative $\left(u_{\varepsilon}\right)_{\varepsilon}$ of classical smooth solutions such that

$$
\begin{gathered}
D_{t}^{2} u_{\varepsilon}(t, x)-\sum_{i=1}^{n} b_{i, \varepsilon}(t) D_{t} D_{x_{i}} u_{\varepsilon}(t, x)-\sum_{i=1}^{n} a_{i, \varepsilon}(t) D_{x_{i}}^{2} u_{\varepsilon}(t, x)=0, \\
u_{\varepsilon}(0, x)=g_{0, \varepsilon}, \\
D_{t} u_{\varepsilon}(0, x)=g_{1, \varepsilon},
\end{gathered}
$$


for suitable embeddings of coefficients and initial data as discussed previously in Case 3. Note that the nets $\left(a_{i, \varepsilon}-a_{i}\right)_{\varepsilon}$ and $\left(b_{i, \varepsilon}-b_{i}\right)_{\varepsilon}$ are converging to 0 in $C\left([0, T] \times \mathbb{R}^{n}\right)$ for $i=1, \ldots, n$ and that $g_{0, \varepsilon}-g_{0}$ and $g_{1, \varepsilon}-g_{1}$ are nets of distributions converging to 0 as well. As in (ii) we can write

$$
\begin{aligned}
& D_{t}^{2}\left(u_{\varepsilon}-\widetilde{u}\right)(t, x)-\sum_{i=1}^{n} b_{i, \varepsilon}(t) D_{t} D_{x_{i}}\left(u_{\varepsilon}-\widetilde{u}\right)(t, x) \\
& \quad-\sum_{i=1}^{n} a_{i, \varepsilon}(t) D_{x_{i}}^{2}\left(u_{\varepsilon}-\widetilde{u}\right)(t, x)=n_{\varepsilon}(t, x), \\
& u_{\varepsilon}(0, x)-\widetilde{u}(0, x)=g_{0, \varepsilon}-g_{0}, \\
& D_{t} u_{\varepsilon}(0, x)-D_{t} \widetilde{u}(0, x)=g_{1, \varepsilon}-g_{1},
\end{aligned}
$$

where $\left(n_{\varepsilon}\right)_{\varepsilon}$ is converging to 0 in $C\left([0, T] ; \mathcal{D}_{(s)}^{\prime}\left(\mathbb{R}^{n}\right)\right)$ and the nets $g_{0, \varepsilon}-g_{0}$ and $g_{1, \varepsilon}-g_{1}$ are converging to 0 in the sense of distributions. From the estimate (54) we deduce that $\widetilde{V}-V_{\varepsilon} \rightarrow 0$ in $C\left([0, T] ; \mathcal{D}_{(s)}^{\prime}\left(\mathbb{R}^{n}\right)\right)$ or in other words that $u_{\varepsilon} \rightarrow \widetilde{u}$ in $C\left([0, T] ; \mathcal{D}_{(s)}^{\prime}\left(\mathbb{R}^{n}\right)\right)$. Analogously, this result is not affected by changing the representative $\left(u_{\varepsilon}\right)_{\varepsilon}$ of $u \in \mathcal{G}\left([0, T] ; \mathcal{G}^{s}\left(\mathbb{R}^{n}\right)\right)$.

Acknowledgments C. Garetto and M. Ruzhansky would like to thank Ferruccio Colombini for a discussion and remarks.

Open Access This article is distributed under the terms of the Creative Commons Attribution License which permits any use, distribution, and reproduction in any medium, provided the original author(s) and the source are credited.

\section{References}

1. Benmeriem, K., Bouzar, C.: Generalized Gevrey ultradistributions. N. Y. J. Math. 15, 37-72 (2009)

2. Bernardi, E., Parenti, C., Parmeggiani, A.: The Cauchy problem for hyperbolic operators with double characteristics in presence of transition. Commun. Partial Differ. Equ. 37(7), 1315-1356 (2012)

3. BRONŠTEIN, M.D.: Smoothness of roots of polynomials depending on parameters. Sibirsk. Mat. Zh. 20(3), 493-501, 690 (1979)

4. BRonšTEǏN, M.D.: The Cauchy problem for hyperbolic operators with characteristics of variable multiplicity. Tr. Moskov. Mat. Obshch. 41, 83-99 (1980)

5. Cicognani, M., Colombini, F.: A well-posed Cauchy problem for an evolution equation with coefficients of low regularity. J. Differ. Equ. 254(8), 3573-3595 (2013)

6. Colombini, F., De Giorgi, E., Spagnolo, S.: Sur les équations hyperboliques avec des coefficients qui ne dépendent que du temps. Ann. Sc. Norm. Sup. Pisa Cl. Sci. (4) 6(3), 511-559 (1979)

7. Colombini, F., Del Santo, D., Kinoshita, T.: Well-posedness of the Cauchy problem for a hyperbolic equation with non-Lipschitz coefficients. Ann. Sc. Norm. Super. Pisa Cl. Sci. (5) 1(2), 327-358 (2002)

8. Colombini, F., Del Santo, D., Reissig, M.: On the optimal regularity of coefficients in hyperbolic Cauchy problems. Bull. Sci. Math. 127(4), 328-347 (2003) 
9. Colombini, F., Jannelli, E., Spagnolo, S.: Well-posedness in the Gevrey classes of the Cauchy problem for a nonstrictly hyperbolic equation with coefficients depending on time. Ann. Sc. Norm. Sup. Pisa Cl. Sci. (4) 10(2), 291-312 (1983)

10. Colombini, F., Jannelli, E., Spagnolo, S.: Nonuniqueness in hyperbolic Cauchy problems. Ann. Math. (2) 126(3), 495-524 (1987)

11. Colombini, F., Kinoshita, T.: On the Gevrey well posedness of the Cauchy problem for weakly hyperbolic equations of higher order. J. Differ. Equ. 186(2), 394-419 (2002)

12. Colombini, F., OrRù, N., Pernazza, L.: On the regularity of the roots of hyperbolic polynomials. Isr. J. Math. 191(2), 923-944 (2012)

13. Colombini, F., Spagnolo, S.: An example of a weakly hyperbolic Cauchy problem not well posed in $C^{\infty}$. Acta Math. 148, 243-253 (1982)

14. D'Ancona, P., Spagnolo, S.: Quasi-symmetrization of hyperbolic systems and propagation of the analytic regularity. Boll. Unione Mat. Ital. Sez. B Artic. Ric. Mat. (8) 1(1), 169-185 (1998)

15. Gelfand, I.M.: Some questions of analysis and differential equations. Am. Math. Soc. Transl. (2). 26, 201-219 (1963)

16. Garetto, C., Ruzhansky, M.: On the well-posedness of weakly hyperbolic equations with time-dependent coefficients. J. Differ. Equ. 253(5), 1317-1340 (2012)

17. Garetto, C., Ruzhansky, M.: Weakly hyperbolic equations with non-analytic coefficients and lower order terms. Math. Ann. 357(2), 401-440 (2013)

18. Garetto, C., Ruzhansky, M.: A note on weakly hyperbolic equations with analytic principal part. J. Math. Anal. Appl. 412(1), 1-14 (2014)

19. Hörmann, G., DE Hoop, M.V.: Microlocal analysis and global solutions of some hyperbolic equations with discontinuous coefficients. Acta Appl. Math. 67(2), 173-224 (2001)

20. Hörmann, G., DE Hoop, M.V.: Detection of wave front set perturbations via correlation: foundation for wave-equation tomography. Appl. Anal. 81(6), 1443-1465 (2002)

21. Hurd, A.E., SATtinger, D.H.: Questions of existence and uniqueness for hyperbolic equations with discontinuous coefficients. Trans. Am. Math. Soc. 132, 159-174 (1968)

22. Kato, T.: Perturbation Theory for Linear Operators. Classics in Mathematics. Springer, Berlin (1995). Reprint of the 1980 edition

23. Kinoshita, T., Spagnolo, S.: Hyperbolic equations with non-analytic coefficients. Math. Ann. 336(3), 551-569 (2006)

24. Lafon, F., ObergugGenberger, M.: Generalized solutions to symmetric hyperbolic systems with discontinuous coefficients: the multidimensional case. J. Math. Anal. Appl. 160(1), 93-106 (1991)

25. Marsan, D., Bean, C.J.: Multiscaling nature of sonic velocities and lithology in the upper crystalline crust: evidence from the KTB main borehole. Geophys. Res. Lett. 26, 275-278 (1999)

26. Nishitani, T.: Sur les équations hyperboliques à coefficients höldériens en $t$ et de classe de Gevrey en x. Bull. Sci. Math. (2) 107(2), 113-138 (1983)

27. Nicola, F., Rodino, L.: Global Pseudo-Differential Calculus on Euclidean Spaces. Pseudo-Differential Operators. Theory and Applications, vol. 4. Birkhäuser, Basel (2010)

28. Oberguggenberger, M.: Multiplication of Distributions and Applications to Partial Differential Equations. Pitman Research Notes in Mathematics Series, vol. 259. Longman Scientific \& Technical, Harlow (1992)

29. Parenti, C., Parmeggiani, A.: On the Cauchy problem for hyperbolic operators with double characteristics. Commun. Partial Differ. Equ. 34(7-9), 837-888 (2009)

30. Schwartz, L.: Sur l'impossibilité de la multiplication des distributions. C. R. Acad. Sci. Paris 239, 847-848 (1954)

31. Teofanov, N.: Modulation spaces, Gelfand-Shilov spaces and pseudodifferential operators. Sampl. Theory Signal Image Process 5(2), 225-242 (2006) 
Department of Mathematical Sciences, Loughborough University, Loughborough, Leicestershire LE11 3TU, UK. e-mail: c.garetto@lboro.ac.uk and

Department of Mathematics, Imperial College London, 180 Queen's Gate, London SW7 2AZ, UK.

e-mail: m.ruzhansky@imperial.ac.uk

(Received January 4, 2014 / Accepted December 2, 2014)

Published online December 20, 2014 - (C) The Author(s) (2014)

This article is published with open access at Springerlink.com 